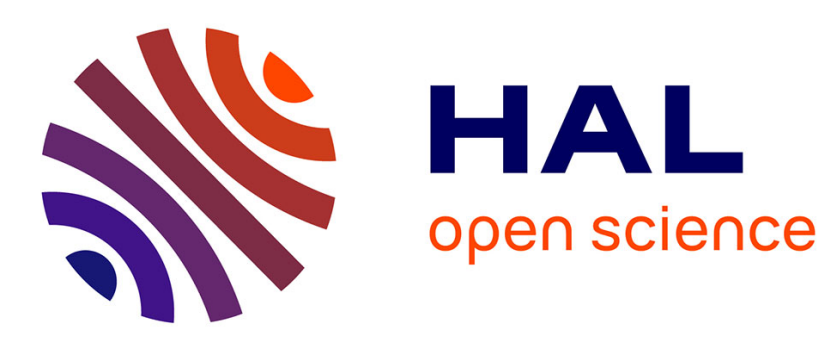

\title{
On the Morita Reduced Versions of Skew Group Algebras of Path Algebras
}

\author{
Patrick Le Meur
}

\section{To cite this version:}

Patrick Le Meur. On the Morita Reduced Versions of Skew Group Algebras of Path Algebras. Quarterly Journal of Mathematics, 2020, 71 (3), 10.1093/qmathj/haaa014 . hal-02361334v2

\section{HAL Id: hal-02361334 \\ https://hal.science/hal-02361334v2}

Submitted on 16 Feb 2023

HAL is a multi-disciplinary open access archive for the deposit and dissemination of scientific research documents, whether they are published or not. The documents may come from teaching and research institutions in France or abroad, or from public or private research centers.
L'archive ouverte pluridisciplinaire HAL, est destinée au dépôt et à la diffusion de documents scientifiques de niveau recherche, publiés ou non, émanant des établissements d'enseignement et de recherche français ou étrangers, des laboratoires publics ou privés. 


\title{
ON THE MORITA REDUCED VERSIONS OF SKEW GROUP ALGEBRAS OF PATH ALGEBRAS
}

\author{
PATRICK LE MEUR
}

\begin{abstract}
Let $R$ be the skew group algebra of a finite group acting on the path algebra of a quiver. This article develops both theoretical and practical methods to do computations in the Morita reduced algebra associated to $R$. Reiten and Riedtmann proved that there exists an idempotent $e$ of $R$ such that the algebra $e R e$ is both Morita equivalent to $R$ and isomorphic to the path algebra of some quiver which was described by Demonet. This article gives explicit formulas for the decomposition of any element of $e$ Re as a linear combination of paths in the quiver described by Demonet. This is done by expressing appropriate compositions and pairings in a suitable monoidal category which takes into account the representation theory of the finite group.
\end{abstract}

\section{INTRODUCTION}

In [13], Reiten and Riedtmann initiated the investigation of the skew group algebras of Artin algebras from the viewpoint of homological dimensions and representation theory. Their article was followed up by many research works illustrating the following principle: Artin algebras share many properties, whether of homological or of representation theoretic nature, with their associated skew group algebras. For instance, see [12] for interactions with Koszul duality; [4, 11] for interactions with piecewise hereditary algebras; and $[14,3]$ for interactions with preprojective algebras and McKay correspondence.

Let $\mathbb{k}$ be an algebraically closed field, $Q$ be a finite quiver that may have oriented cycles, and $G$ be a finite group with order not divisible by $\operatorname{char}(\mathbb{k})$ and acting on $\mathbb{k} Q$ by algebra automorphisms in such a way that both the set of (primitive idempotents of) vertices and the vector subspace generated by the arrows are stabilised by this action. Following [13], the skew group algebra $\mathbb{k} Q * G$ is hereditary.

While Reiten and Riedtmann described a quiver whose path algebra is Morita equivalent to $\mathbb{k} Q * G$ when $G$ is cyclic, the description of such a quiver in general is recent. In [3], Demonet described an idempotent $\tilde{e}$ of $\mathbb{k} Q * G$ and a quiver $Q_{G}$ such that $\tilde{e} \cdot(\mathbb{k} Q * G) \cdot \tilde{e}$ is Morita equivalent to $\mathbb{k} Q * G$ and isomorphic to $\mathbb{k} Q_{G}$. This, however, does not yield a completely described isomorphism, which might be source of trouble. Here is a situation taken from Calabi-Yau algebras and where such trouble may occur. Following [10], if $W$ is a $G$-invariant potential on $Q$, then $G$ acts on the Ginzburg dg algebra $\mathcal{A}(Q, W)$, defined in [6], and $\mathcal{A}(Q, W) * G$ is Morita equivalent to $\mathcal{A}\left(Q_{G}, W_{G}\right)$, where $W_{G}$ is the potential on $Q_{G}$ which, as a linear combination of oriented cycles, corresponds to $\tilde{e} \cdot W \cdot \tilde{e}$ under any chosen isomorphism $\mathbb{k} Q_{G} \rightarrow \tilde{e} \cdot(\mathbb{k} Q * G) \cdot \tilde{e}$. The problem here is that describing that linear combination explicitly is not easy. Up to now, the only fairly general explicit descriptions of $W_{G}$ are due to Amiot and Plamondon [1] when $G$ is of order 2 and to Giovannini and Pasquali [8] when $G$ is cyclic, the stabiliser of each vertex is either trivial or the whole group, and every cycle appearing in $W$ goes through fixed

Date: June 30, 2020.

2010 Mathematics Subject Classification. Primary 16S35; Secondary 16W22, 20C15, 18D10.

Key words and phrases. Path algebra; skew group algebra; monoidal category; intertwiner. 
vertices only (or, through vertices with trivial stabilisers only, respectively) as soon as it goes through two of them; during the revision of the present article, Giovannini, Pasquali and Plamondon presented [9] an extension of the latter description to the case where $G$ is finite and abelian.

This raises the question of decomposing explicitly the elements of $\tilde{e} \cdot\left(\mathbb{k}_{Q} * G\right) \cdot \tilde{e}$ as linear combinations of paths in $Q_{G}$ under an isomorphism $\mathbb{k} Q_{G} \rightarrow \tilde{e} \cdot(\mathbb{k} Q * G) \cdot \tilde{e}$.

Demonet's description of $Q_{G}$ involves the representation theory of the stabilisers of the vertices of $Q$. It is hence expectable that answering the above-mentioned question should involve representation theory. Answers of this kind already exist in particular cases where $Q$ has only one vertex. Here is an example due to Ginzburg [6]. He proved that, if $G$ is a finite subgroup of $\mathrm{SL}_{3}(\mathbb{C})$, then $\mathbb{C}[x, y, z] * G$ is Calabi-Yau in dimension 3. This was done by taking $Q$ to be the quiver with one vertex and three loops $x, y$, and $z$, by taking $W=x y z-x z y$, and by expressing $W_{G}$ in terms of the monoidal category of finite dimensional representations of $G$. See [2] for a generalisation to $\mathbb{C}\left[x_{1}, \ldots, x_{n}\right] * G$ where $G$ is a finite subgroup of $\mathrm{SL}_{n}(\mathbb{C})$, in which case $Q$ is the quiver with one vertex and $n$ loops. Note that this generalisation is (detailed and) successfully applied by Giovannini in [7] to find subgroups $\Gamma$ of $\mathrm{SL}_{n+1}(\mathbb{C})$ such that $\mathbb{C}\left[x_{1}, \ldots, x_{n+1}\right] * \Gamma$ is Morita equivalent to the $(n+1)$-preprojective algebra of an $n$-representation finite algebra.

This article hence gives explicit formulas for the decomposition of the elements of $\tilde{e} \cdot(\mathbb{k} Q * G) \cdot \tilde{e}$ as linear combinations of paths in $Q_{G}$ under the isomorphism $\mathbb{k} Q_{G} \rightarrow \tilde{e} \cdot(\mathbb{k} Q * G) \cdot \tilde{e}$. These formulas are obtained by translating explicitly the result of natural operations and pairings in the monoidal category $\left(\bmod \left(A^{e}\right), \otimes_{A}\right)$ of finite dimensional $A$-bimodules, where $A$ is the direct product of the group algebras of the stabilisers of the vertices of $Q$.

On one hand, these formulas provide an interpretation of the coefficients of these linear combinations in terms of the representation theory of the stabilisers of the vertices of $Q$. On the other hand, they are explicit enough to be implemented by a computer or a human being able to manipulate the irreducible representations of the involved groups. In specific cases, for instance when the action of the group elements transforms arrows to scalar multiples of arrows and the stabilisers are cyclic, these formulas simplify in a combinatorial way. Finally, these formulas give a complete solution to the problem of computing a potential $W_{G}$ on $Q_{G}$ such that the Ginzburg dg algebra $\mathcal{A}\left(Q_{G}, W_{G}\right)$ is Morita equivalent to $\mathcal{A}(Q, W) * G$, for any given $G$-invariant potential $W$ on $Q$.

\section{Definitions AND MAIN RESUlts}

Throughout the article, $\mathbb{k}$ denotes an algebraically closed field. For all finite dimensional algebras $\Lambda$, their categories of finite dimensional left modules are denoted by $\bmod (\Lambda)$.

This section presents the main results of this text as well as the needed definitions and notation. These definitions are illustrated with a running example throughout the section. A full application of the main results is presented on an example in section 2. The remaining sections are devoted to the proofs of these results.

Setting 1.1. Let $Q_{0}, S, G$, and $M$ be as follows.

- $Q_{0}$ is a finite set and $S$ is the semi-simple $\mathbb{k}$-algebra $\mathbb{k} Q_{0}$.

- $G$ is a finite group with order not divisible by $\operatorname{char}(\mathbb{k})$ and acting on $Q_{0}$.

- $M$ is a finite dimensional $S$-bimodule, considered as a collection of vector spaces $\left({ }_{i} M_{j}\right)_{(i, j) \in Q_{0}^{2}}$, endowed with an action of $G$ written exponentially and such that ${ }^{g}\left({ }_{i} M_{j}\right)={ }_{g \cdot i} M_{g \cdot j}$ for all $i, j \in Q_{0}$ and $g \in G$. 
The choice of a basis of ${ }_{i} M_{j}$, for all $i, j \in Q_{0}$ determines a quiver $Q$ such that $T_{S}(M) \simeq \mathbb{k} Q$ as $\mathbb{k}$-algebras. All finite quivers arise in this way.

The actions of $G$ on $S$ and $M$ induce an action on $T_{S}(M)$ by algebra automorphisms such that ${ }^{g}\left(m_{1} \otimes \cdots \otimes m_{n}\right)={ }^{g} m_{1} \otimes \cdots \otimes{ }^{g} m_{n}$ for all $g \in G$ and $m_{1}, \ldots, m_{n} \in M$. Recall that the skew group algebra $T_{S}(M) * G$ is the $\mathbb{k}$-algebra with underlying vector space $T_{S}(M) \otimes G$, where the tensor $u \otimes v$ is denoted by $u * v$ for all $u \in T_{S}(M)$ and $v \in \mathbb{k} G$, and with product such that $(u * g) \cdot\left(u^{\prime} * g^{\prime}\right)=\left(u \cdot{ }^{g} u^{\prime}\right) * g g^{\prime}$ for all $u, u^{\prime} \in T_{S}(M)$ and $g, g^{\prime} \in G$. It is customary to consider $T_{S}(M)$ and $\mathbb{k}_{k} G$ as subalgebras of $T_{S}(M) * G$ in the canonical way.

Notation 1.2. The following data is used throughout the article.

- $\left[G \backslash Q_{0}\right]$ denotes a complete set of representatives of the orbits in $Q_{0}$.

- For all $i \in Q_{0}$,

- $G_{i}$ denotes the stabiliser of $i$ and $e_{i}$ denotes the corresponding primitive idempotent of $S$,

- $\left[G / G_{i}\right]$ denotes a complete set of representatives of cosets modulo $G_{i}$,

- $\operatorname{irr}\left(G_{i}\right)$ denotes a complete set of representatives of the isomorphism classes of the irreducible representations of $G_{i}$,

- and $\varepsilon_{U}$ denotes a primitive idempotent of $\mathbb{k}_{k} G_{i}$ such that $U \simeq \mathbb{k} G_{i} \cdot \varepsilon_{U}$ in $\bmod \left(\mathbb{k}_{k} G_{i}\right)$, for all $U \in \operatorname{irr}\left(G_{i}\right)$.

- $\tilde{e}$ denotes the idempotent $\sum_{(i, U)} e_{i} * \varepsilon_{U}$ of $T_{S}(M) * G$, where $i$ runs through $\left[G \backslash Q_{0}\right]$ and $U$ runs through $\operatorname{irr}\left(G_{i}\right)$.

Example 1.3. Let $Q$ be the quiver

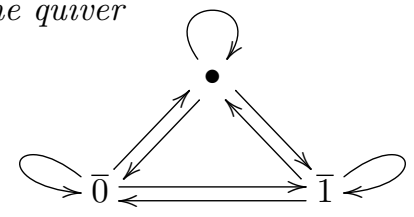

where $\overline{0}$ and $\overline{1}$ denote the classes in $\mathbb{Z} / 2 \mathbb{Z}$. For all vertices $i, j$ of $Q$, the arrow from $i$ to $j$ is denoted by $x_{i, j}$. Let $G$ be the group $\left\langle\tau \mid \tau^{2}\right\rangle$ of order 2 . The actions of $G$ on $Q$ and on $M$ are assumed to be the ones such that

$$
\tau \cdot \bullet=\bullet, \quad \tau \cdot \overline{0}=\overline{1},
$$

and, for all vertices $i, j$ of $Q$,

$$
{ }^{\tau} x_{i, j}=-x_{\tau \cdot i, \tau \cdot j} \cdot
$$

- Let $\left[G \backslash Q_{0}\right]$ be $\{\bullet, \overline{0}\}$. Note that $G_{\bullet}=\{\operatorname{Id}, \tau\}$ and $G_{\overline{0}}=\{\operatorname{Id}\}$.

- Let $\left[G / G_{\bullet}\right]$ and $\left[G / G_{\overline{0}}\right]$ be $\{\operatorname{Id}\}$ and $\{\mathrm{Id}, \tau\}$, respectively.

- For all $k \in \mathbb{Z} / 2 \mathbb{Z}$, denote by $\varepsilon_{k}$ the idempotent $\frac{1}{2}\left(\mathrm{Id}+(-1)^{k} \tau\right)$; denote by $\rho_{k}$ the irreducible representation $\mathbb{k} \cdot \varepsilon_{k}$ of $G$.

- Let $\operatorname{irr}\left(G_{\bullet}\right)$ and $\operatorname{irr}\left(G_{\overline{0}}\right)$ be $\left\{\rho_{k} \mid k \in \mathbb{Z} / 2 \mathbb{Z}\right\}$ and $\left\{\mathbb{k}_{\mathrm{k}} \cdot \mathrm{Id}\right\}$, respectively.

In this situation, $\tilde{e}=e_{\bullet} * \varepsilon_{\overline{0}}+e_{\bullet} * \varepsilon_{\overline{1}}+e_{\overline{0}} * \mathrm{Id}$.

1.1. Demonet's quiver of the Morita reduction. Demonet proved in [3] that the skew group algebra $T_{S}(M) * G$ is Morita equivalent to the path algebra of a quiver as follows.

Setting 1.4. Let $Q_{G}$ be a quiver with the following properties.

- The vertices are the couples $(i, U)$, where $i \in\left[G \backslash Q_{0}\right]$ and $U \in \operatorname{irr}\left(G_{i}\right)$.

- For all vertices $(i, U)$ and $(j, V)$, the arrows $(i, U) \rightarrow(j, V)$ form a basis over $\mathbb{k}$ of $\operatorname{Hom}_{\mathbb{k} G_{i}}(U, M(i, j ; V))$, here $M(i, j ; V)=\oplus_{y \in\left[G / G_{j}\right] i} M_{y \cdot j} \otimes_{\mathbb{k}} y V$, where $y V$ stands for $y \otimes_{\mathbb{k}} V \subset \mathbb{k}_{k} G \otimes_{\mathbb{k}} V$, see Definition 1.8 for details. 
Example 1.5. This is the continuation of Example 1.3. In order to describe a quiver $Q_{G}$ such as in Setting 1.4, Table 1 escribes the $G_{i}$-module $M(i, j ; U)$ for all possible $i, j, U$.

\begin{tabular}{lcc}
\hline$M(i, j ; U)$ & Basis over $\mathbb{k}$ & Action of $G_{i}$ \\
\hline$M\left(\bullet, \bullet ; \rho_{k}\right)$ & $x_{\bullet, \bullet} \otimes \varepsilon_{k}$ & $\tau\left(x_{\bullet, \bullet} \otimes \varepsilon_{k}\right)=(-1)^{k+1} x_{\bullet \bullet \bullet} \otimes \varepsilon_{k}$ \\
$M(\bullet \overline{0} ; \mathbb{k} \cdot \mathrm{Id})$ & $\left(x_{\bullet, k} \otimes \tau^{k}\right)_{k \in \mathbb{Z} / 2 \mathbb{Z}}$ & $\tau\left(x_{\bullet, k} \otimes \tau^{k}\right)=-x_{\bullet, k+\overline{1}} \otimes \tau^{k+1}$ \\
$M\left(\overline{0}, \bullet ; \rho_{k}\right)$ & $x_{\overline{0}, \bullet} \otimes \varepsilon_{k}$ & Trivial \\
$M(\overline{0}, \overline{0} ; \mathbb{k} \cdot \mathrm{Id})$ & $\left(x_{\overline{0}, k} \otimes \tau^{k}\right)_{k \in \mathbb{Z} / 2 \mathbb{Z}}$ & Trivial \\
\hline
\end{tabular}

TABLE 1 . The $G_{i}$-modules $M(i, j ; U)(k \in \mathbb{Z} / 2 \mathbb{Z})$.

For all $k \in \mathbb{Z} / 2 \mathbb{Z}$, denote by $a_{k}$ and $b_{k}$ the following respective morphisms of $G_{\bullet}$-modules

$$
\begin{array}{llll}
\rho_{k+\overline{1}} \longrightarrow M\left(\bullet, \bullet ; \rho_{k}\right) & \rho_{k} \longrightarrow M(\bullet, \overline{0} ; \mathbb{k} \cdot \mathrm{Id}) \\
\varepsilon_{k+\overline{1}} \longmapsto x_{\bullet, \bullet} \otimes \varepsilon_{k} & \varepsilon_{k} \longmapsto \frac{1}{2}\left(x_{\bullet}, \overline{0} \otimes \mathrm{Id}+(-1)^{k+\overline{1}} x_{\bullet, \overline{1}} \otimes \tau\right)
\end{array}
$$

and denote by $c_{k}$ and $d_{k}$ the following respective morphisms of $G_{\overline{0}}$-modules

$$
\begin{aligned}
\mathbb{k} \cdot \mathrm{Id} & \longrightarrow M\left(\overline{0}, \bullet ; \rho_{k}\right) & \mathbb{k} \cdot \mathrm{Id} & \longrightarrow M\left(\overline{0}, \overline{0} ; \mathbb{k}_{k} \cdot \mathrm{Id}\right) \\
\mathrm{Id} & \longmapsto x_{\overline{0}, \bullet} \otimes \varepsilon_{k} & \text { Id } & \longmapsto
\end{aligned}
$$

Then, $Q_{G}$ may be taken equal to

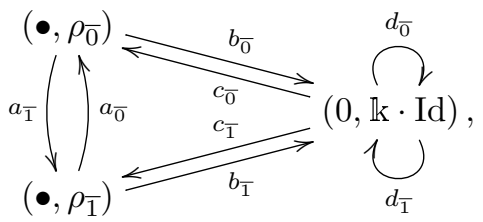

Demonet's result may be reformulated as follows, see Section 5 for more details.

Theorem $1.6([3])$. The $\mathbb{k}$-algebras $\mathbb{k}_{k} Q_{G}$ and $T_{S}(M) * G$ are Morita equivalent. Besides, assuming that $U=\mathbb{k}_{i} \cdot \varepsilon_{U}$ for all vertices $(i, U)$ of $Q_{G}$, then there exists an isomorphism of algebras

$$
\mathbb{k}_{k} Q_{G} \stackrel{\sim}{\longrightarrow} \tilde{e} \cdot\left(T_{S}(M) * G\right) \cdot \tilde{e}
$$

which maps every arrow $f:(i, U) \rightarrow(j, V)$ of $Q_{G}$ to $f\left(\varepsilon_{U}\right)$.

1.2. Monoidal categories as a toolbox for intertwiners. The present article introduces a $\mathbb{k}$-algebra, called the algebra of intertwiners relative to $M$ and denoted by $\mathcal{I}_{n t w}$, and an isomorphism of $\mathbb{k}_{\text {-algebras }} \mathbb{k}_{k} Q_{G} \rightarrow \mathcal{I}_{n t w}{ }^{\text {op }}$ together with explicit formulas decomposing any given element of $\mathcal{I}$ ntw in the basis consisting of the images of the paths in $Q_{G}$ under $\mathbb{k}_{Q_{G}} \rightarrow \mathcal{I}_{\text {ntw }}{ }^{\text {op }}$. See Theorem 1.25 for details. Composing this isomorphism with the inverse of (1.1.1) results in a $\mathbb{k}$-algebra isomorphism $\tilde{e} \cdot\left(T_{S}(M) * G\right) \cdot \tilde{e} \rightarrow \mathcal{I n t w}^{\text {op }}$. A description of it is given in Theorem 1.27 hence providing a decomposition, under (1.1.1), of any element of $\tilde{e} \cdot\left(T_{S}(M) * G\right) \cdot \tilde{e}$ in the basis of $\mathbb{k}_{k} Q_{G}$ consisting of the paths in $Q_{G}$. These decompositions involve non-degenerate pairings between intertwiners relative to $M$ and intertwiners relative to the dual vector space $M^{*}$. Both the product in $\mathcal{I}$ ntw and the pairings are explicit reformulations of natural operations and pairings in a suitable monoidal category.

Remark $1.7([5])$. Let $(\mathcal{C}, \otimes)$ be a monoidal $\mathbb{k}$-linear category with finite dimensional morphism spaces. 
(1) Given objects $X, Y, U, V$, and $W$ of $\mathcal{C}$, and following the ideas presented in [6, Subsection 4.4] for the monoidal categories of finite subgroups of $\mathrm{SL}_{3}(\mathbb{C})$, there is a natural operation between morphisms $U \rightarrow X \otimes V$ and $V \rightarrow Y \otimes W$

$$
\begin{array}{ccc}
\mathcal{C}(U, X \otimes V) \times \mathcal{C}(V, Y \otimes W) & \longrightarrow & \mathcal{C}(U, X \otimes Y \otimes W) \\
\left(f, f^{\prime}\right) & \longmapsto & \left(X \otimes f^{\prime}\right) \circ f .
\end{array}
$$

(2) Given an object $X$ of $\mathcal{C}$, recall that a left dual of $X$ is an object $X^{\prime}$ of $\mathcal{C}$ together with an adjunction $(X \otimes-) \vdash\left(X^{\prime} \otimes-\right)$ of endofunctors of $\mathcal{C}$ ([5, Definition 2.10.1, Proposition 2.10.8]). If $X$ has a left dual, then there exists a non-degenerate pairing, for all objects $U$ and $V$ of $\mathcal{C}$ such that $\mathcal{C}(U, U)=\mathbb{k} \cdot \operatorname{Id}_{U}$,

$$
\begin{array}{ccc}
\langle-\mid-\rangle: \mathcal{C}(U, X \otimes V) \otimes \mathcal{C}\left(V, X^{\prime} \otimes U\right) & \longrightarrow & \mathbb{k} \\
f \otimes \phi & \longmapsto & \langle f \mid \phi\rangle
\end{array}
$$

such that $\langle f \mid \phi\rangle \cdot \operatorname{Id}_{U}=\bar{\phi} \circ f$, where $\bar{\phi} \in \mathcal{C}(X \otimes V, U)$ is adjoint to $\phi$; because $\mathcal{C}(U, U)=\mathbb{k} \cdot \operatorname{Id}_{U}$, a scalar is associated to every endomorphism of $U$, and hence to $\bar{\phi} \circ f$, which makes it possible to define a pairing with values in $\mathbb{k}$. When $\mathcal{C}$ is a multitensor category this pairing is just a combination of the adjunction $(X \otimes-) \vdash\left(X^{\prime} \otimes-\right)$ and the non-degenerate pairing $\mathcal{C}(U, X \otimes V) \otimes \mathcal{C}(X \otimes V, U) \rightarrow \mathbb{k}$ which takes into account $U$ being simple in $\mathcal{C}$ (see [5, Example 7.9.14]).

The monoidal category $\mathcal{C}$ considered here is the category $\bmod \left(A^{e}\right)$ of finite dimensional $A$-bimodules with tensor product being $\otimes_{A}$, where $A$ is as follows,

$$
A=\mathbb{k} \times \Pi_{i \in Q_{0}} \mathbb{k}_{k} G_{i} .
$$

Note that $A$ is a semi-simple $\mathbb{k}$-algebra; accordingly, any object $X$ of $\mathcal{C}$ has a left dual given by the dual vector space $X^{*}$. The isolated factor $\mathbb{k}$ in the definition of $A$ is included so that $\mathcal{C}$ contains $\bmod \left(\mathbb{k}_{i}\right)$ as a full additive subcategory, yet not as a monoidal subcategory, for all $i \in Q_{0}$. The reason for this inclusion is the following, at the same time it clarifies the role of $\mathcal{C}$. The text manipulates morphism spaces of the shape

(1.2.2) $\operatorname{Hom}_{\mathbb{k} G_{i_{0}}}\left(U, e_{i_{0}}\left(M \otimes_{\mathbb{k}} \mathbb{k} G\right) e_{i_{1}} \otimes_{\mathbb{k} G_{i_{1}}} \cdots \otimes_{\mathbb{k} G_{i_{n-1}}} e_{i_{n-1}}\left(M \otimes_{\mathbb{k}} \mathbb{k} G\right) e_{i_{n}} \otimes_{\mathbb{k} G_{i_{n}}} V\right)$

where $i_{0}, \ldots, i_{n}$ are vertices of $Q$ whereas $U \in \bmod \left(\mathbb{k} G_{i_{0}}\right)$ and $V \in \bmod \left(\mathbb{k}_{k} G_{i_{n}}\right)$; note that each one of $e_{i_{t-1}}\left(M \otimes_{\mathbb{k}} \mathbb{k} G\right) e_{i_{t}}$ (for $1 \leqslant t \leqslant n$ ) is an object of $\mathcal{C}$, and so are $U$ and $V$ because of the isolated factor $\mathbb{k}$ in $A$; the above-mentioned morphism space is hence equal to

$$
\mathcal{C}\left(U, e_{i_{0}}\left(M \otimes_{\mathbb{k}} \mathbb{k} G\right) e_{i_{1}} \otimes \cdots \otimes e_{i_{n-1}}\left(M \otimes_{\mathbb{k}} \mathbb{k} G\right) e_{i_{n}} \otimes V\right)
$$

and it is manipulated in this text using the tools recalled in Remark 1.7.

1.3. The interwiners. Here are the base ingredients needed to define $\mathcal{I}$ ntw.

Definition 1.8. Let $n$ be a non-negative integer. Let $\underline{i}=i_{0}, \ldots, i_{n}$ be a sequence in $Q_{0}$. Let $V \in \bmod \left(\mathbb{k}_{i_{n}}\right)$.

(1) For all sequences $\underline{y}=y_{1}, \ldots, y_{n}$, where $y_{t} \in\left[G / G_{i_{t}}\right]$ for all $t \in\{1, \ldots, n\}$, let $M_{\underline{y}}(\underline{i} ; V)$ be the following vector space,

$M_{\underline{y}}(\underline{i} ; V)={ }_{i_{0}} M_{y_{1} \cdot i_{1}} \otimes_{\mathrm{k} y_{1} \cdot i_{1}} M_{y_{1} y_{2} \cdot i_{2}} \otimes_{\mathrm{k}} \cdots \otimes_{\mathrm{k} y_{1} \cdots y_{n-1} \cdot i_{n-1}} M_{y_{1} \cdots y_{n} \cdot i_{n}} \otimes_{\mathrm{k}} y_{1} \cdots y_{n} V$, where $y_{1} \cdots y_{n} V$ stands for the vector subspace $y_{1} \cdots y_{n} \otimes_{\mathbb{k}} V$ of $\mathbb{k}_{k} G \otimes_{\mathbb{k}} V$. 
(2) Define the $\mathbb{k}_{k} G_{i_{0}}$-module $M(\underline{i} ; V)$ as follows. Its underlying vector space is

$$
\bigoplus_{\underline{y}} M_{\underline{y}}(\underline{i} ; V)
$$

where $y$ runs through all possible sequences such as in (1). For all $g \in G_{i_{0}}$, the action of $g$ is written exponentially and defined as follows. For all $y$, there exist a unique sequence $\underline{y}^{\prime}=y_{1}^{\prime}, \ldots, y_{n}^{\prime}$, where $y_{t}^{\prime} \in\left[G / G_{i_{t}}\right]$ for all $t \in\{1, \ldots, n\}$, and a unique $h \bar{\in} G_{i_{n}}$, such that

- $g y_{1} \cdots y_{t} G_{i_{t}}=y_{1}^{\prime} \cdots y_{t}^{\prime} G_{i_{t}}$ for all $t \in\{1, \ldots, n\}$,

- and $g y_{1} \cdots y_{n}=y_{1}^{\prime} \cdots y_{n}^{\prime} h$;

then, the action of $g$ transforms $M_{\underline{y}}(\underline{i} ; V)$ into $M_{\underline{y}^{\prime}}(\underline{i} ; V)$ and, for all $m_{1} \otimes$ $\cdots \otimes m_{n} \otimes y_{1} \cdots y_{n} v \in M_{\underline{y}}(\underline{i} ; V)$,

$$
{ }^{g}\left(m_{1} \otimes \cdots \otimes m_{n} \otimes y_{1} \cdots y_{n} v\right)={ }^{g} m_{1} \otimes \cdots \otimes{ }^{g} m_{n} \otimes y_{1}^{\prime} \cdots y_{n}^{\prime}{ }^{h} v .
$$

This does define a finite dimensional $\mathbb{k}_{i_{0}}$-module. If $n=0$, then $M(\underline{i} ; V)=V$. In the monoidal category $\left(\bmod \left(A^{e}\right), \otimes_{A}\right)$ considered previously, $M(\underline{i} ; V)$ is isomorphic to $M(\underline{i}) \otimes_{A} V$ where $M(\underline{i})$ is a $\mathbb{k} G_{i_{0}}-\mathbb{k}_{k} G_{i_{n}}$-bimodule defined by $M$ and $\underline{i}$, see (4.1.1) for the definition of $M(\underline{i})$ and (4.1.7) for the isomorphism.

Note that, in the previous definition, if $U=\mathbb{k} G_{i_{0}} \cdot \varepsilon_{U}$ and $V=\mathbb{k} G_{i_{n}} \cdot \varepsilon_{V}$ for primitive idempotents $\varepsilon_{U} \in \mathbb{k}_{k} G_{i_{0}}$ and $\varepsilon_{V} \in \mathbb{k} G_{i_{n}}$, then $M(\underline{i} ; U)$ is a $\mathbb{k}_{k} G_{i_{0}}$ submodule of $T_{S}(M) * G$.

Example 1.9. This is the continuation of Example 1.5. Let $k \in \mathbb{Z} / 2 \mathbb{Z}$. Then $M\left(\bullet, \overline{0}, \overline{0}, \bullet ; \rho_{k}\right)$ identifies with the $\mathbb{k}_{\mathrm{k}} G_{\bullet}$-submodule of $T_{S}(M) * G$ whose underlying

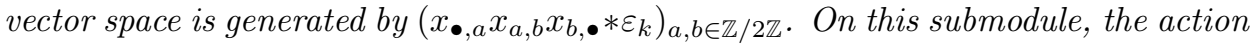
of $\tau$ is such that ${ }^{\tau}\left(x_{\bullet, a} x_{a, b} x_{b, \bullet} * \varepsilon_{k}\right)=(-1)^{k+\overline{1}} x_{\bullet, a+\overline{1}} x_{a+\overline{1}, b+\overline{1}} x_{b+\overline{1}, \bullet} * \varepsilon_{k}$.

The main results of this article are based on a description of the quiver $\mathbb{k}_{Q_{G}}$ in terms of intertwiners.

Definition 1.10. An intertwiner relative to $M$ is a morphism of $\mathbb{k}_{k} G_{i_{0}}$-modules $U \rightarrow M(\underline{i} ; V)$, for some sequence $\underline{i}=i_{0}, \ldots, i_{n}$ in $Q_{0}$ and some modules $U \in$ $\bmod \left(\mathbb{k}_{k} G_{i_{0}}\right)$ and $V \in \bmod \left(\mathbb{k}_{k} G_{i_{n}}\right)$.

Note that, in the previous definition, if $U=\mathbb{k}_{k} G_{i_{0}} \cdot \varepsilon_{U}$ and $V=\mathbb{k}_{k} G_{i_{n}} \cdot \varepsilon_{V}$ for primitive idempotents $\varepsilon_{U} \in \mathbb{k}_{k} G_{i_{0}}$ and $\varepsilon_{V} \in \mathbb{k} G_{i_{n}}$, then all intertwiners $U \rightarrow$ $M(\underline{i} ; V)$ take their values in $T_{S}(M) * G$.

Example 1.11. This is the continuation of Example 1.9. Let $s, t \in \mathbb{Z} / 2 \mathbb{Z}$. For all $a, b \in \mathbb{Z} / 2 \mathbb{Z}$, the following holds true in $M\left(\bullet, \overline{0}, \overline{0}, \bullet ; \rho_{t}\right)$,

$\varepsilon_{s} \cdot\left(x_{\bullet, a} x_{a, b} x_{b, \bullet} \otimes \varepsilon_{t}\right)=\frac{1}{2}\left(x_{\bullet, a} x_{a, b} x_{b, \bullet} \otimes \varepsilon_{t}+(-1)^{s+\overline{1}+t} x_{\bullet, a+\overline{1}} x_{a+\overline{1}, b+\overline{1}} x_{b+\overline{1}, \bullet} \otimes \varepsilon_{t}\right)$.

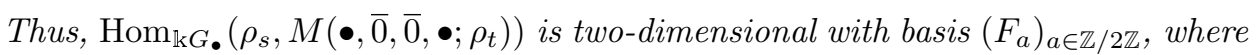
$F_{a}$ denotes the following intertwiner relative to $M$, for all $a \in \mathbb{Z} / 2 \mathbb{Z}$,

$$
\begin{aligned}
& \rho_{s} \longrightarrow M\left(\bullet, \overline{0}, \overline{0}, \bullet ; \rho_{t}\right) \\
& \varepsilon_{s} \longmapsto \frac{1}{2}\left(x_{\bullet, \overline{0}} x_{\overline{0}, a} x_{a, \bullet} \otimes \varepsilon_{t}+(-1)^{s+\overline{1}+t} x_{\bullet, \overline{1}} x_{\overline{1}, a+\overline{1}} x_{a+\overline{1}, \bullet} \otimes \varepsilon_{t}\right) .
\end{aligned}
$$

The main purpose of this article is to provide explicit formulas in the main theorems. These formulas use the following notation.

Notation 1.12. Let $\underline{i}=i_{0}, \ldots, i_{n}$ be a sequence in $Q_{0}$ where $n \geqslant 1$. Let $U \in$ $\bmod \left(\mathbb{k}_{k} G_{i_{0}}\right)$ and $V \in \bmod \left(\mathbb{k}_{k} G_{i_{n}}\right)$. Let $f \in \operatorname{Hom}_{\mathbb{k} G_{i_{0}}}(U, M(\underline{i} ; V))$. For all $u \in U$, write the decomposition of $f(u)$ along (1.3.1) as follows

$$
f(u)=\sum_{\underline{y}} f_{\underline{y}}(u)
$$


and, for all sequences $y=y_{1}, \ldots, y_{n}$, where $y_{t} \in\left[G / G_{i_{t}}\right]$ for all $t \in\{1, \ldots, n\}$, write symbolically the element $f_{y}(u)$ of $M_{y}(\underline{i} ; V)$ as follows, with the sum sign omitted,

$$
f_{\underline{y}}(u)=f_{\underline{y}}^{(1)}(u) \otimes \cdots \otimes f_{\underline{y}}^{(n)}(u) \otimes y_{1} \cdots y_{n} f_{\underline{y}}^{(0)}(u) ;
$$

hence, $f_{\underline{y}}^{(t)}(u)$ is meant to lie in $y_{1} \cdots y_{t-1} \cdot i_{t-1} M_{y_{1} \cdots y_{t} \cdot i_{t}}$, for all $t \in\{1, \ldots, n\}$, and $f_{\underline{y}}^{(0)}(u)$ is meant to lie in $V$; as a whole,

$$
f(u)=\sum_{\underline{y}} f_{\underline{y}}^{(1)}(u) \otimes \cdots \otimes f_{\underline{y}}^{(n)}(u) \otimes y_{1} \cdots y_{n} f_{\underline{y}}^{(0)}(u) .
$$

1.4. The algebra of intertwiners. Now here is the operation to be used as the multiplication in $\mathcal{I}$ ntw.

Definition 1.13. Let $\underline{i}^{\prime \prime}=i_{0}, \ldots, i_{m+n}$ be a sequence in $Q_{0}$, where $m, n \geqslant 1$. Denote the sequences $i_{0}, \ldots, i_{m}$ and $i_{m}, i_{m+1}, \ldots, i_{m+n}$ by $\underline{i}$ and $\underline{i}^{\prime}$, respectively. Let $U \in \bmod \left(\mathbb{k}_{k} G_{i_{0}}\right), V \in \bmod \left(\mathbb{k} G_{i_{m}}\right)$, and $W \in \bmod \left(\mathbb{k}_{k} G_{i_{m+n}}\right)$. For all $f \in$ $\operatorname{Hom}_{\mathbb{k} G_{i_{0}}}(U, M(\underline{i} ; V))$ and $f^{\prime} \in \operatorname{Hom}_{\mathbb{k} G_{i_{m}}}\left(V, M\left(\underline{i}^{\prime} ; W\right)\right)$, define a $\mathbb{k}$-linear mapping

$$
f^{\prime} \circledast f: U \rightarrow M\left(\underline{i^{\prime \prime}} ; W\right)
$$

by

$$
f^{\prime} \circledast f=\sum_{\underline{y^{\prime \prime}}}\left(f^{\prime} \circledast f\right)_{\underline{y^{\prime \prime}}},
$$

where

- $y^{\prime \prime}$ runs through all sequences $y_{1}, \ldots, y_{m+n}$ with $y_{t} \in\left[G / G_{i_{t}}\right]$ for all $t$

- and $\left(f^{\prime} \circledast f\right)_{\underline{y}^{\prime \prime}}$ is the mapping $U \rightarrow M_{\underline{y}^{\prime \prime}}\left(\underline{i}^{\prime \prime} ; W\right)$ such that, for all $u \in U$,

$$
\begin{aligned}
\left(f^{\prime} \circledast f\right)_{\underline{y^{\prime \prime}}}(u)= & f_{\underline{y}}^{(1)}(u) \otimes \cdots \otimes f_{\underline{y}}^{(m)}(u) \otimes \\
& y_{1} \cdots y_{m}\left(f_{\underline{y^{\prime}}}^{\prime(1)}\left(f_{\underline{y}}^{(0)}(u)\right)\right) \otimes \cdots \otimes y_{1} \cdots y_{m}\left(f_{\underline{y^{\prime}}}^{\prime(n)}\left(f_{\underline{y}}^{(0)}(u)\right)\right) \otimes \\
& y_{1} \cdots y_{m+n} f_{\underline{y^{\prime}}}^{\prime(0)}\left(f_{\underline{y}}^{(0)}(u)\right),
\end{aligned}
$$

where $\underline{y}=y_{1}, \ldots, y_{m}$ and $\underline{y^{\prime}}=y_{m+1}, \ldots, y_{m+n}$.

Note that, in the previous definition, if $U=\mathbb{k}_{k} G_{i_{0}} \cdot \varepsilon_{U}, V=\mathbb{k}_{k} G_{i_{m}} \cdot \varepsilon_{V}$, and $W=$ $\mathbb{k} G_{i_{m+n}} \cdot \varepsilon_{W}$ for primitive idempotents $\varepsilon_{U} \in \mathbb{k} G_{i_{0}}, \varepsilon_{V} \in \mathbb{k} G_{i_{m}}$, and $\varepsilon_{W} \in \mathbb{k} G_{i_{m+n}}$, then

$$
\left(f^{\prime} \circledast f\right)\left(\varepsilon_{U}\right)=f\left(\varepsilon_{U}\right) \cdot f^{\prime}\left(\varepsilon_{v}\right),
$$

where the product on the right-hand side is taken in $T_{S}(M) * G$.

Remark 1.14. The definition of $\circledast$ is technical. Actually, it is an explicit reformulation of a simple operation in the monoidal category $\left(\bmod \left(A^{e}\right), \otimes_{A}\right)$. More precisely, using the comment that follows Definition 1.8,

- first, $M\left(\underline{i}^{\prime \prime}\right) \simeq M(\underline{i}) \otimes_{A} M\left(\underline{i}^{\prime}\right)$, see Lemma 4.2 ;

- next, if $M(\underline{i} ; V), M\left(\underline{i}^{\prime} ; W\right)$, and $M\left(\underline{i}^{\prime \prime} ; W\right)$ are identified with $M(\underline{i}) \otimes_{A} V$, $M\left(\underline{i}^{\prime}\right) \otimes_{A} W$, and $M\left(\underline{i}^{\prime \prime}\right) \otimes_{A} W$, respectively, then $\circledast$ is an explicit reformulation of the operation introduced in part (1) of Remark 1.7, see Lemma 4.4 for a precise statement and proof.

In view of the previous remark, $\circledast$ does yield intertwiners, see Lemma 4.4, and it is associative, see Proposition 4.5. 
Definition 1.15. The algebra of intertwiners relative to $M$ is denoted by $\mathcal{I}$ ntw and defined by

$$
\text { Intw }=\left(\bigoplus_{n, \underline{i}, U, V} \operatorname{Hom}_{\mathbb{k} G_{i_{0}}}(U, M(\underline{i} ; V)), \circledast\right),
$$

where $n$ runs through all non negative integers, $\underline{i}=i_{0}, \ldots, i_{n}$ runs through all sequences in $\left[G \backslash Q_{0}\right]$, and $U$ and $V$ run through $\operatorname{irr}\left(G_{i_{0}}\right)$ and $\operatorname{irr}\left(G_{i_{n}}\right)$, respectively.

It is hence possible to associate an element of $\mathcal{I}$ ntw to every path in $Q_{G}$.

Notation 1.16. For all paths in $Q_{G}$

$$
\gamma:\left(i_{0}, U_{0}\right) \stackrel{f_{1}}{\longrightarrow}\left(i_{1}, U_{1}\right) \rightarrow \cdots \rightarrow\left(i_{n-1}, U_{n-1}\right) \stackrel{f_{n}}{\longrightarrow}\left(i_{n}, U_{n}\right),
$$

denote by $f_{\gamma}$ the following element of $\mathcal{I}$ ntw lying in $\operatorname{Hom}_{\mathbb{k} G_{i_{0}}}\left(U_{0}, M\left(i_{0}, \ldots, i_{n} ; U_{n}\right)\right)$,

$$
f_{\gamma}=f_{n} \circledast f_{n-1} \circledast \cdots \circledast f_{1} .
$$

1.5. Dual intertwiners and their pairing with intertwiners. As stated below, see Theorem 1.25, assigning $f_{\gamma}$ to $\gamma$ for every path $\gamma$ in $Q_{G}$ yields an isomorphism of algebras from $\mathbb{k}_{k} Q_{G}$ to $\mathcal{I n t w}^{\text {op }}$. The purpose of this article is to explain how to decompose any element of $\mathcal{I}$ ntw as a linear combination of the $f_{\gamma}$ 's. This involves non-degenerate pairings between certain spaces of intertwiners relative to $M$ and to $M^{*}$, respectively. Here, $M^{*}$ denotes the dual vector $\operatorname{space} \operatorname{Hom}_{\mathbb{k}}(M, \mathbb{k})$, it has a structure of $S$-bimodule such that ${ }_{i} M_{j}^{*}=\left({ }_{j} M_{i}\right)^{*}$ for all $i, j \in Q_{0}$, and $G$ acts on $M^{*}$ in such a way that, for all $\varphi \in M^{*}$ and $g \in G$,

$$
{ }^{g} \varphi=\varphi\left({ }^{g^{-1} \bullet}\right) \text {. }
$$

This action is such that ${ }^{g}\left({ }_{i} M_{j}^{*}\right)={ }_{g \cdot i} M_{g \cdot j}^{*}$ for all $i, j \in Q_{0}$ and $g \in G$. Hence, the previous considerations may be applied to $M^{*}$ instead of to $M$.

Example 1.17. This is the continuation of Example 1.11. For all vertices $i, j$ of $Q$, the vector space ${ }_{i} M_{j}^{*}$ is one-dimensional; denote by $x_{i, j}^{\prime}$ its base element, dual to the base element $x_{j, i}$ of ${ }_{j} M_{i}$; Hence, ${ }^{\tau} x_{i, j}^{\prime}=-x_{\tau(i), \tau(j)}^{\prime}$. After replacing $M$ by $M^{*}$, the considerations of Example 1.11 yield that $\operatorname{Hom}_{\mathbb{k} G}\left(\rho_{t}, M^{*}\left(\bullet, \overline{0}, \overline{0}, \bullet ; \rho_{s}\right)\right)$ is

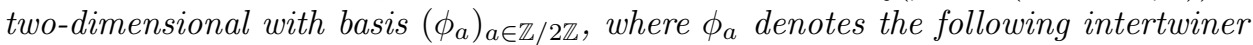
relative to $M^{*}$, for all $a \in \mathbb{Z} / 2 \mathbb{Z}$,

$$
\begin{aligned}
& \rho_{t} \longrightarrow M^{*}\left(\bullet, \overline{0}, \overline{0}, \bullet ; \rho_{s}\right) \\
& \left.\varepsilon_{t} \longmapsto x_{\bullet, \overline{0}} x_{\overline{0}, a} x_{a, \bullet} \otimes \varepsilon_{s}+(-1)^{t+\overline{1}+s} x_{\bullet, \overline{1}} x_{\overline{1}, a+\overline{1}} x_{a+\overline{1}, \bullet} \otimes \varepsilon_{s}\right) .
\end{aligned}
$$

Notation 1.18. Let $\underline{i}=i_{0}, \ldots, i_{n}$ be a sequence in $Q_{0}$, where $n \geqslant 0$. Let $U \in$ $\bmod \left(\mathbb{k}_{k} G_{i_{0}}\right)$ and $V \in \bmod \left(\mathbb{k}_{k} G_{i_{n}}\right)$.

(1) Denote by $\underline{i}^{o}$ the sequence $i_{n}, i_{n-1}, \ldots, i_{0}$.

(2) Let $\varphi \in \operatorname{Hom}_{\mathbb{k} G_{i_{n}}}\left(V, M^{*}\left(\underline{i}^{o}, U\right)\right)$. Proceeding similarly as done in Notation 1.12 ,

- denote by $\varphi^{\underline{x}}$ the composition of $\varphi$ with the canonical projection $M^{*}\left(\underline{i}^{o} ; U\right) \rightarrow M_{x}^{*}\left(\underline{i}^{o} ; U\right)$, for all sequences $\underline{x}=x_{n-1}, x_{n-2}, \ldots, x_{0}$ where $x_{t} \in\left[G / G_{i_{t}}\right]$ for all $t \in\{0, \ldots, n-1\}$,

- and for all such $\underline{x}$ and all $v \in V$, write symbolically the element $\varphi^{\underline{x}}(v)$ of $M_{\underline{x}}^{*}\left(\underline{i}^{o} ; U\right)$ as follows,

$$
\varphi^{\underline{x}}(v)=\varphi_{(n)}^{\frac{x}{(n)}}(v) \otimes \cdots \otimes \varphi_{(1)}^{\frac{x}{(1)}}(v) \otimes x_{n-1} \cdots x_{0} \varphi_{(0)}^{\frac{x}{(0)}}(v) .
$$


Hence, $\varphi_{(t)}^{\frac{x}{(t)}}(v)$ is meant to lie in $\left(x_{n-1} x_{n-2} \cdots x_{t}\right) \cdot i_{t} M_{\left(x_{n-1} x_{n-2} \cdots x_{t-1}\right) \cdot i_{t-1}}^{*}$ for all $t \in\{1, \ldots, n\}$ and $\varphi_{(0)}^{\underline{x}}(v)$ is meant to lie in $U$. As a whole,

$$
\varphi(v)=\sum_{\underline{x}} \varphi_{(n)}^{\underline{x}}(v) \otimes \cdots \otimes \varphi_{(1)}^{\underline{x}}(v) \otimes x_{n-1} \cdots x_{1} \varphi_{(0)}^{\underline{x}}(v) .
$$

Here is an illustration of this notation.

Example 1.19. This is the continuation of Example 1.17. Assume that $n=2$ and $\underline{i}=\overline{0}, \overline{0}, \bullet$. Hence $\underline{i}^{o}=\bullet, \overline{0}, \overline{0}$. The underlying vector space of the $\mathbb{k}_{\bullet} G_{\bullet}$-module $M^{*}\left(\underline{i}^{o} ; \mathbb{k} \cdot \mathrm{Id}\right)$ is freely generated by $\left(x_{\bullet, a}^{\prime} x_{a, b}^{\prime} \otimes \tau^{b}\right)_{a, b \in \mathbb{Z} / 2 \mathbb{Z} \cdot}$.

Let $a, b, s \in \mathbb{Z} / 2 \mathbb{Z}$. Denote by $\varphi$ the intertwiner $\rho_{s} \rightarrow M^{*}\left(\underline{i}^{o} ; \mathbb{k} \cdot \mathrm{Id}\right)$ such that $\varphi\left(\varepsilon_{s}\right)=\varepsilon_{s} \cdot\left(x_{\bullet, a}^{\prime} x_{a, b}^{\prime} \otimes \tau^{b}\right)$, that is $\varphi\left(\varepsilon_{s}\right)=\frac{1}{2} x_{\bullet, a}^{\prime} x_{a, b}^{\prime} \otimes \tau^{b}+\frac{(-1)^{s}}{2} x_{\bullet, a+1}^{\prime} x_{a+\overline{1}, b+\overline{1}}^{\prime} \otimes$ $\tau^{b+\overline{1}}$. On one hand, $x_{\bullet, a}^{\prime} x_{a, b}^{\prime}=x_{\bullet, \tau^{a} \cdot \overline{0}}^{\prime} x_{\tau^{a} \cdot \overline{0}, \tau^{a} \tau^{b-a}, \overline{0}}^{\prime}$; Hence

$$
\left\{\begin{aligned}
\varphi^{\tau^{a}, \tau^{b-a}}\left(\varepsilon_{s}\right) & =\frac{1}{2} x^{\prime}, a x_{a, b}^{\prime} \otimes \tau^{b} \\
\varphi_{(2)}^{\tau^{a}, \tau^{b-a}}\left(\varepsilon_{s}\right) \otimes \varphi_{(1)}^{\tau^{a}, \tau^{b-a}}\left(\varepsilon_{s}\right) \otimes \varphi_{(0)}^{\tau^{a}, \tau^{b-a}}\left(\varepsilon_{s}\right) & =\frac{1}{2} x_{\bullet, a}^{\prime} \otimes x_{a, b}^{\prime} \otimes \mathrm{Id} .
\end{aligned}\right.
$$

On the other hand, $x_{\bullet, a+\overline{1}}^{\prime} x_{a+\overline{1}, b+\overline{1}}^{\prime}=x_{\bullet, \tau^{a+\overline{1}} \cdot \overline{0}}^{\prime} x_{\tau^{a+1} \cdot \overline{0}, \tau^{a+\overline{1}} \tau^{b-a \cdot \overline{0}}}^{\prime}$; Hence

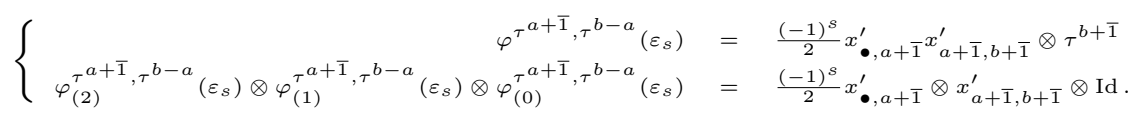

In view of description of $\varphi\left(\varepsilon_{s}\right)$ made initially, all the remaining terms of the sum of (1.5.1) are zero.

The above mentioned pairings are described explicitly as follows.

Proposition 1.20. Let $\underline{i}=i_{0}, \ldots, i_{n}$ be a sequence in $Q_{0}$, where $n \geqslant 0$. Let $U \in \bmod \left(\mathbb{k} G_{i_{0}}\right)$ and $V \in \bmod \left(\mathbb{k} G_{i_{n}}\right)$. Assume that $U$ is simple. There exists a non-degenerate pairing

$$
(-\mid-): \operatorname{Hom}_{\mathrm{k} G_{i_{0}}}(U, M(\underline{i} ; V)) \otimes_{\mathrm{k}} \operatorname{Hom}_{\mathrm{k} G_{i_{n}}}\left(V, M^{*}\left(\underline{i}^{o} ; U\right)\right) \rightarrow \mathbb{k}
$$

with the following property. For all $f \in \operatorname{Hom}_{\mathbb{k} G_{i_{0}}}(U, M(\underline{i} ; V))$ and for all $\varphi \in$ $\operatorname{Hom}_{k G_{i_{n}}}\left(V, M^{*}\left(\underline{i}^{o} ; U\right)\right)$, the scalar $(f \mid \varphi)$ is such that, for all $u \in U$, the element $(f \mid \varphi)$ u of $U$ is equal to

$$
\sum_{\underline{y}} \prod_{t=1}^{n} \varphi_{(t)}^{\underline{x}}\left(f_{\underline{y}}^{(0)}(u)\right)\left({ }^{\left(y_{1} \cdots y_{n}\right)^{-1}} f_{\underline{y}}^{(t)}(u)\right)^{h_{0}}\left(\varphi_{(0)}^{\underline{x}}\left(f_{\underline{y}}^{(0)}(u)\right)\right),
$$

where $\underline{y}=y_{1}, \ldots, y_{n}$ runs through all sequences with $y_{t} \in\left[G / G_{i_{t}}\right]$ for all $t$ and $\underline{x}=x_{n-1}, \ldots, x_{0}$ and $h_{0}$ are uniquely determined by the following conditions:

(a) $x_{t} \in\left[G / G_{i_{t}}\right]$ for all $t$, and $h_{0} \in G_{i_{0}}$.

(b) $\left(y_{t} \cdots y_{n}\right)^{-1} \in x_{n-1} x_{n-2} \cdots x_{t-1} G_{i_{t-1}}$ for all $t \in\{1, \ldots, n\}$.

(c) $\left(y_{1} \cdots y_{n}\right)^{-1}=x_{n-1} x_{n-2} \cdots x_{0} h_{0}$.

In view of computations, here is a useful explanation regarding $\underline{x}$ and $h_{0}$ in the above proposition. For all sequences $\underline{y}=y_{1}, \ldots, y_{n}$ in $G$ such that $y_{t} \in\left[G / G_{i_{t}}\right]$ for all $t \in\{1, \ldots, n\}$ and for all non zero tensors

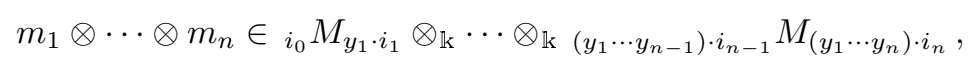

there exists a unique sequence $\underline{x}=x_{n-1}, x_{n-2}, \ldots, x_{0}$ in $G$ such that $x_{t} \in\left[G / G_{i_{t}}\right]$ for all $t \in\{0, \ldots, n-1\}$ and

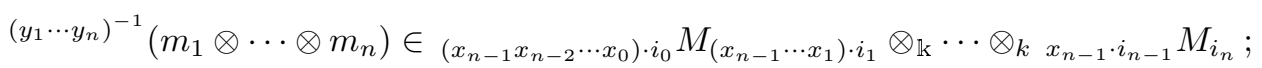
besides, $\left(x_{n-1} x_{n-2} \cdots x_{0}\right)^{-1}\left(y_{1} \cdots y_{n}\right)^{-1} \in G_{i_{0}}$. This sequence $\underline{x}$ is the one in the statement of Proposition 1.20 in which $h_{0}=\left(x_{n-1} x_{n-2} \cdots x_{0}\right)^{-1}\left(y_{1} \cdots y_{n}\right)^{-1}$. 
When the action of $G$ on $M$ is such that arrows of $Q$ are transformed into scalar multiples of arrows of $Q$, the expression (1.5.2) of $(-\mid-)$ simplifies to (2.1.3). The following example illustrates the computation of $(-\mid-)$ in this particular situation.

Example 1.21. This is the continuation of Example 1.17. Let $s, t \in \mathbb{Z} / 2 \mathbb{Z}$. Let $a, b \in \mathbb{Z} / 2 \mathbb{Z}$. Consider $F_{a}: \rho_{s} \rightarrow M\left(\bullet, \overline{0}, \overline{0}, \bullet ; \rho_{t}\right)$ and $\phi_{b}: \rho_{t} \rightarrow M\left(\bullet, \overline{0}, \overline{0}, \bullet ; \rho_{s}\right)$ such as in Examples 1.11 and 1.17, respectively.

In view of (2.1.3), $\left(F_{a} \mid \phi_{b}\right)$ is a sum of products of scalars, the sum is indexed by the paths in $Q$ that appear in the expression of $F_{a}\left(\varepsilon_{s}\right)$. For all such paths $\gamma$, the scalars forming the corresponding product appear in Table 2 with bold face. For convenience, this table presents additional data, using the following notation, see Subsection 2.1 for more details. For a given path $\gamma$ in $Q$ :

- $\alpha_{\gamma}$ denotes the coefficient of $\gamma \otimes \varepsilon_{t}$ in $F_{a}\left(\varepsilon_{s}\right)$;

- $y_{1}, y_{2}, y_{3}$ is the sequence of $G$ such that $y_{1}, y_{2} \in\left[G / G_{\overline{0}}\right], y_{3} \in\left[G / G_{\bullet}\right]$ and $\gamma$ is a path of the shape $\bullet \rightarrow y_{1} \cdot \overline{0} \rightarrow y_{1} y_{2} \cdot \overline{0} \rightarrow y_{1} y_{2} y_{3} \cdot \bullet$;

- $\left(y_{1} y_{2} y_{3}\right)^{-1}(\gamma)$ denotes the path of $Q$ and $\chi_{\left(y_{1} y_{2} y_{3}\right)^{-1}, \gamma}$ denotes the scalar

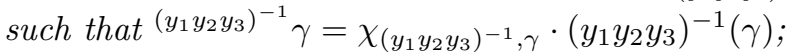

- $\left(y_{1} y_{2} y_{3}\right)^{-1}(\gamma)^{*}$ denotes the element of the basis of $T_{S}\left(M^{*}\right)$ dual to the basis of $T_{S}(M)$ consisting of the paths of $Q$, associated to $\left(y_{1} y_{2} y_{3}\right)^{-1}(\gamma)$;

- $\beta_{\left(y_{1} y_{2} y_{3}\right)^{-1}(\gamma)^{*}}$ denotes the coefficient of $\left(y_{1} y_{2} y_{3}\right)^{-1}(\gamma)^{*}$ in $\phi_{b}\left(\varepsilon_{t}\right)$;

- $\chi_{\rho_{s}}$ denotes the character of $\rho_{s}$.

Thus,

$$
\left(F_{a} \mid \phi_{b}\right)= \begin{cases}\frac{(-1)^{(s+\overline{1}) a} \cdot\left(1+(-1)^{t}\right)}{2} & \text { if } a=b \\ 0 & \text { if not. }\end{cases}
$$

\begin{tabular}{|c|c|c|}
\hline$\gamma$ & $x_{\bullet, \overline{0}} x_{\overline{0}, a} x_{a, \bullet}$ & $x_{\bullet, \overline{1}} x_{\overline{1}, a+\overline{1}} x_{a+\overline{1}, \bullet}$ \\
\hline$\alpha_{\gamma}$ & $\frac{1}{2}$ & $\frac{(-1)^{\mathbf{s}+\overline{1}+t}}{2}$ \\
\hline $\begin{array}{l}y_{1}, y_{2}, y_{3} \\
\left(y_{1} y_{2} y_{3}\right)^{-1} \gamma\end{array}$ & $\begin{array}{c}\text { Id, } \tau^{a}, \text { Id } \\
(-1)^{a} x_{\bullet,-a} x_{-a, \overline{0}} x_{\overline{0}, \bullet}\end{array}$ & $\begin{array}{c}\tau, \tau^{a}, \operatorname{Id} \\
(-1)^{a+\overline{1}} x_{\bullet,-a} x_{-a, \overline{0}} x_{\overline{0}, \bullet}\end{array}$ \\
\hline $\begin{array}{l}\chi\left(\mathbf{y}_{1} \mathbf{y}_{\mathbf{2}} \mathbf{y}_{\mathbf{3}}\right)^{-1}, \gamma \\
\left(y_{1} y_{2} y_{3}\right)^{-1}(\gamma)^{*}\end{array}$ & $x_{\bullet, 0}^{\prime} x_{\overline{0},-a}^{(-1)^{\mathbf{a}}} x_{-a, \bullet}^{\prime}$ & $\begin{array}{c}(-\mathbf{1})^{\mathbf{a}+\overline{\mathbf{1}}} \\
x_{\bullet, \overline{0}}^{\prime} x_{\overline{0},-a}^{\prime} x_{-a, \bullet}^{\prime}\end{array}$ \\
\hline$\beta_{\left(\mathbf{y}_{1} \mathbf{y}_{2} \mathbf{y}_{3}\right)^{-1}(\gamma)^{*}}$ & $\begin{cases}1 & \text { if } b=-a \\
0 & \text { if not }\end{cases}$ & $\begin{array}{l}1 \text { if } b=-a \\
0 \text { if not }\end{array}$ \\
\hline $\begin{array}{l}x_{2}, x_{1}, x_{0}, h_{0} \\
\chi_{\rho_{\mathbf{s}}}\left(\mathbf{h}_{\mathbf{0}}\right)\end{array}$ & $\begin{array}{c}\mathrm{Id}, \tau^{-a}, \mathrm{Id}, \tau^{-a} \\
(-\mathbf{1})^{\mathbf{s a}}\end{array}$ & $\begin{array}{l}\mathrm{Id}, \tau^{-a}, \mathrm{Id}, \tau^{-a-\overline{1}} \\
\quad(-\mathbf{1})^{\mathbf{s}(\mathbf{a}+\overline{\mathbf{1}})}\end{array}$ \\
\hline $\begin{array}{l}\text { contribution of } \gamma \text { to } \\
\left(F_{a} \mid \phi_{b}\right)\end{array}$ & $\begin{array}{ll}\frac{(-1)^{a(s+\overline{1})}}{2} & \text { if } b=-a \\
0 & \text { if not }\end{array}$ & $\begin{array}{ll}\frac{(-1)^{(s+\overline{1}) a+t}}{2} & \text { if } b \\
0 & \text { if } n\end{array}$ \\
\hline
\end{tabular}

TABLE 2. The scalars appearing in the expression (2.1.3) of $\left(F_{a} \mid \phi_{b}\right)$

Remark 1.22. Like the operation $\circledast$, the pairing $(-\mid-)$ has a simple interpretation in the monoidal category $\left(\bmod \left(A^{e}\right), \otimes_{A}\right)$. Indeed,

- first, $M(\underline{i})$ admits $M^{*}\left(\underline{i}^{o}\right)$ as a left dual, see (4.1.5);

- next, if $M(\underline{i} ; V)$ and $M^{*}\left(\underline{i}^{o} ; U\right)$ are identified with $M(\underline{i}) \otimes_{A} V$ and $M^{*}\left(\underline{i}^{o}\right) \otimes_{A}$ $U$, respectively, then $(-\mid-)$ is an explicit reformulation of $\langle-\mid-\rangle$ as introduced in part (2) of Remark 1.7, see (4.3.8).

Using $(-\mid-)$ yields intertwiners relative to $M^{*}$ associated with the $f_{\gamma}$ 's for paths $\gamma$ in $Q_{G}$. 
Notation 1.23. Let $Q_{G}$ be such as in Setting 1.4.

(1) Let $f \in(i, U) \rightarrow(j, V)$ be an arrow of $Q_{G}$. Using the basis of the vector space $\operatorname{Hom}_{\mathbb{k} G_{i}}(U, M(i, j ; V))$ consisting of the arrows $(i, U) \rightarrow(j, V)$ of $Q_{G}$ and using the associated (-|-)-dual basis of $\operatorname{Hom}_{\mathbb{k} G_{j}}\left(V, M^{*}(j, i ; U)\right)$, denote by $f^{\vee}$ the dual element associated with $f$. In other words, $f^{\vee}$ lies in $\operatorname{Hom}_{\mathbb{k} G_{j}}\left(V, M^{*}(j, i ; U)\right)$ and, for all arrows $f^{\prime}:(i, U) \rightarrow(j, V)$ of $Q_{G}$,

$$
\left(f^{\prime} \mid f^{\vee}\right)= \begin{cases}1 & \text { if } f^{\prime}=f \\ 0 & \text { otherwise. }\end{cases}
$$

(2) For all paths $\gamma$ in $Q_{G}$

$$
\gamma:\left(i_{0}, U_{0}\right) \stackrel{f_{1}}{\longrightarrow}\left(i_{1}, U_{1}\right) \rightarrow \cdots \rightarrow\left(i_{n-1}, U_{n-1}\right) \stackrel{f_{n}}{\longrightarrow}\left(i_{n}, U_{n}\right),
$$

denote by $\varphi_{\gamma}$ the following intertwiner

$$
\varphi_{\gamma}=f_{1}^{\vee} \circledast f_{2}^{\vee} \circledast \cdots \circledast f_{n}^{\vee} \in \operatorname{Hom}_{\mathbb{k} G_{i_{n}}}\left(U_{n}, M^{*}\left(i_{n}, i_{n-1}, \ldots, i_{0} ; U_{0}\right)\right) .
$$

Example 1.24. This is the continuation of Example 1.21, it illustrates Notation 1.23.

Let $k \in \mathbb{Z} / 2 \mathbb{Z}$. Denote by $a_{k}^{\prime}$ and $b_{k}^{\prime}$ the following respective intertwiners relative to $M^{*}$

$$
\begin{aligned}
& \rho_{k} \quad \longrightarrow \quad M^{*}\left(\bullet, \bullet ; \rho_{k+\overline{1}}\right) \quad \mathbb{k} \cdot \mathrm{Id} \quad \longrightarrow \quad M^{*}\left(\overline{0}, \bullet ; \rho_{k}\right) \\
& \varepsilon_{k} \longmapsto x_{\bullet, \bullet}^{\prime} \otimes \varepsilon_{k+\overline{1}} \quad \text { Id } \longmapsto x_{\overline{0}, \bullet}^{\prime} \otimes \varepsilon_{k} \text {. }
\end{aligned}
$$

Denote by $c_{k}^{\prime}$ and $d_{k}^{\prime}$ the following respective intertwiners relative to $M^{*}$

$$
\begin{aligned}
& \rho_{k} \longrightarrow M^{*}(\bullet, \overline{0} ; \mathbb{k} \cdot \mathrm{Id}) \quad \mathbb{k} \cdot \mathrm{Id} \longrightarrow M^{*}\left(\overline{0}, \overline{0} ; \mathbb{k}_{k} \cdot \mathrm{Id}\right) \\
& \varepsilon_{k} \longmapsto\left(x_{\bullet, \overline{0}}^{\prime} \otimes \mathrm{Id}+(-1)^{k+\overline{1}} x_{\bullet, \overline{1}}^{\prime} \otimes \tau\right) \quad \text { Id } \longmapsto x_{\overline{0}, k}^{\prime} \otimes \tau^{k} .
\end{aligned}
$$

Then $\left(a_{k} \mid a_{k}^{\prime}\right)=1,\left(b_{k} \mid b_{k}^{\prime}\right)=1,\left(c_{k} \mid c_{k}^{\prime}\right)=1$ and $\left(d_{k} \mid d_{\ell}^{\prime}\right)=\delta_{k, \ell}$ for all $\ell \in \mathbb{Z} / 2 \mathbb{Z}$, where $\delta_{k, \ell}$ stands for the Kronecker symbol. Using (2.1.3), the intermediate steps of the computation of these pairings are given in Tables 3, 4, 5 and 6 (see Example 1.21). In view of the shape of $Q_{G}$, these equalilities entail that $a_{k}^{\vee}=a_{k}^{\prime}, b_{k}^{\vee}=b_{k}^{\prime}$, $c_{k}^{\vee}=c_{k}$ and $d_{k}^{\vee}=d_{k}^{\prime}$.

\begin{tabular}{cccccccccc}
\hline$\gamma$ & $\alpha_{\gamma}$ & $y_{1}$ & ${ }^{y_{1}^{-1}} \gamma$ & $\chi_{\mathbf{y}_{\mathbf{1}}^{-1}, \gamma}$ & $y_{1}^{-1}(\gamma)^{*}$ & $\beta_{\mathbf{y}_{\mathbf{1}}^{-\mathbf{1}}(\gamma)^{*}}$ & $x_{0}, h_{0}$ & $\chi_{\rho_{\mathbf{k}+\overline{\mathbf{1}}}}\left(\mathbf{h}_{\mathbf{0}}\right)$ & contr. of $\gamma$ \\
\hline$x_{\bullet}, \bullet$ & $\frac{1}{\mathbf{2}}$ & Id & $x_{\bullet}, \bullet$ & $\mathbf{1}$ & $x_{\bullet}^{\prime}, \bullet$ & $\mathbf{1}$ & Id, Id & $\mathbf{1}$ & $\mathbf{1}$ \\
\hline \multicolumn{1}{c}{ TABLE 3.} & $\left(a_{k} \mid a_{k}^{\prime}\right)=1$
\end{tabular}

\begin{tabular}{cccccccccc}
\hline$\gamma$ & $\alpha_{\gamma}$ & $y_{1}$ & $y_{1}^{-1} \gamma$ & $\chi_{\mathbf{y}_{\mathbf{1}}^{-1}, \gamma}$ & $y_{1}^{-1}(\gamma)^{*}$ & $\beta_{\mathbf{y}_{\mathbf{1}}^{-\mathbf{1}}(\gamma)^{*}}$ & $x_{0}, h_{0}$ & $\chi_{\rho_{\mathbf{k}}}\left(\mathbf{h}_{\mathbf{0}}\right)$ & contr. of $\gamma$ \\
\hline$x_{\bullet}, \overline{0}$ & $\frac{1}{2}$ & Id & $x_{\bullet}, \overline{0}$ & $\mathbf{1}$ & $x_{\overline{0}}^{\prime}, \bullet$ & $\mathbf{1}$ & $\operatorname{Id}, \mathrm{Id}$ & $\mathbf{1}$ & $\frac{1}{2}$ \\
$x_{\bullet}, \overline{1}$ & $\frac{(-\mathbf{1})^{\mathbf{k}+\overline{\mathbf{1}}}}{\mathbf{2}}$ & $\tau$ & $-{ }^{-x}, \overline{0}$ & $-\mathbf{1}$ & $x_{\overline{0}, \bullet}^{\prime}$, & $\mathbf{1}$ & $\operatorname{Id}, \tau$ & $(-\mathbf{1})^{\mathbf{k}}$ & $\frac{1}{2}$ \\
\hline \multicolumn{8}{c}{ TABLE } & 4. & $\left(b_{k} \mid b_{k}^{\prime}\right)=\frac{1}{2}+\frac{1}{2}=1$
\end{tabular}

\begin{tabular}{cccccccccc}
\hline$\gamma$ & $\alpha_{\gamma}$ & $y_{1}$ & ${ }^{y_{1}^{-1}} \gamma$ & $\chi_{\mathbf{y}_{1}^{-1}, \gamma}$ & $y_{1}^{-1}(\gamma)^{*}$ & $\beta_{\mathbf{y}_{1}^{-1}(\gamma)^{*}}$ & $x_{0}, h_{0}$ & $\chi_{\rho_{\mathbf{k}}}\left(\mathbf{h}_{\mathbf{0}}\right)$ & contr. of $\gamma$ \\
\hline$x_{\overline{0}, \bullet}$ & $\mathbf{1}$ & Id & $x_{\overline{0}, \bullet}$ & $\mathbf{1}$ & $x_{\bullet}^{\prime}, \overline{0}$ & $\mathbf{1}$ & $\operatorname{Id}$, Id & $\mathbf{1}$ & $\frac{1}{\mathbf{2}}$ \\
\hline \multicolumn{8}{c}{ TABLE 5.} & $\left(c_{k} \mid c_{k}^{\prime}\right)=1$
\end{tabular}

Now it is possible to give examples of intertwiners relative to $M^{*}$ and associated to paths in $Q_{G}$. Let $s, t, a \in \mathbb{Z} / 2 \mathbb{Z}$. Denote by $\gamma$ the following path of $Q_{G}$.

$$
\left(\bullet, \rho_{s}\right) \stackrel{b_{s}}{\longrightarrow}(\overline{0}, \mathbb{k} \cdot \mathrm{Id}) \stackrel{d_{a}}{\longrightarrow}\left(\overline{0}, \mathbb{k}_{k} \cdot \mathrm{Id}\right) \stackrel{c_{t}}{\longrightarrow}\left(\bullet, \rho_{t}\right) .
$$




\begin{tabular}{cccccccccc}
\hline$\gamma$ & $\alpha_{\gamma}$ & $y_{1}$ & $y_{1}^{-1} \gamma$ & $\chi_{\mathbf{y}_{1}^{-1}, \gamma}$ & $y_{1}^{-1}(\gamma)^{*}$ & $\beta_{\mathbf{y}_{1}^{-1}(\gamma) *}$ & $x_{0}, h_{0}$ & $\chi_{\mathbf{k} \cdot \text { Id }}\left(\mathbf{h}_{\mathbf{0}}\right)$ & contr. of $\gamma$ \\
\hline$x_{\overline{0}, k}$ & $(-\mathbf{1})^{\mathbf{k}}$ & $\tau^{\mathrm{k}}$ & $(-1)^{k} x_{k, \overline{0}}$ & $(-\mathbf{1})^{\mathbf{k}}$ & $x_{\overline{0}, k}^{\prime}$ & $\delta_{\mathbf{k}, \ell}$ & $\tau^{-k}, \operatorname{Id}$ & $\mathbf{1}$ & $\delta_{\mathbf{k}, \ell}$ \\
\hline \multicolumn{8}{c}{ TABLE } & $6 .\left(d_{k} \mid d_{\ell}^{\prime}\right)=\delta_{k, \ell}$
\end{tabular}

Then $\varphi_{\gamma}=b_{s}^{\vee} \circledast d_{a}^{\vee} \circledast c_{t}^{\vee}=b_{s}^{\prime} \circledast d_{a}^{\prime} \circledast c_{t}^{\prime}$. This is the intertwiner $\rho_{t} \rightarrow M^{*}\left(\bullet, \overline{0}, \overline{0}, \bullet ; \rho_{s}\right)$ such that (see (1.4.2))

$$
\begin{aligned}
\varphi_{\gamma}\left(\varepsilon_{t}\right) & =c_{t}^{\prime}\left(\varepsilon_{t}\right) \cdot d_{a}^{\prime}(\mathrm{Id}) \cdot b_{s}^{\prime}(\mathrm{Id}) \\
& =\left(x_{\bullet}^{\prime} \otimes \mathrm{Id}+(-1)^{t+\overline{1}} x_{\bullet, \overline{1}}^{\prime} \otimes \tau\right) \cdot\left(x_{\overline{0}, a}^{\prime} \otimes \tau^{a}\right) \cdot\left(x_{\overline{0}, \bullet}^{\prime} \otimes \varepsilon_{s}\right) \\
& =(-1)^{a(s+\overline{1})}\left(x_{\bullet}^{\prime} x_{\overline{0}, a}^{\prime} x_{a, \bullet}^{\prime} \otimes \varepsilon_{s}+(-1)^{t+\overline{1}+s} x_{\bullet, \overline{1}}^{\prime} x_{\overline{1}, a+\overline{1}}^{\prime} x_{a+\overline{1}, \bullet}^{\prime} \otimes \varepsilon_{s}\right) .
\end{aligned}
$$

Thus, using the notation introduced in Example 1.17, $\varphi_{\gamma}=(-1)^{a(s+\overline{1})} \phi_{a}$.

1.6. The comparison of the algebra of intertwiners with the Morita reduction. Now, here is the result comparing $\mathbb{k} Q_{G}$ and $\mathcal{I}$ ntw.

Theorem 1.25. Keep Settings 1.1 and 1.4.

(1) Assigning $f_{\gamma}$ to every path $\gamma$ in $Q_{G}$ yields an isomorphism of algebras

$$
\mathbb{k} Q_{G} \stackrel{\sim}{\rightarrow} \mathcal{I n t w}^{\text {op }} \text {. }
$$

(2) For all $f \in \mathcal{I}$ tw, say lying in $\operatorname{Hom}_{\mathrm{k} G_{i_{0}}}\left(U_{0}, M\left(i_{0}, \ldots, i_{n} ; U_{n}\right)\right)$,

$$
f=\sum_{\gamma}\left(f \mid \varphi_{\gamma}\right) f_{\gamma},
$$

where $\gamma$ runs through all paths of $Q_{G}$ of the shape $\left(i_{0}, U_{0}\right) \rightarrow\left(i_{1}, \bullet\right) \rightarrow$ $\cdots \rightarrow\left(i_{n-1}, \bullet\right) \rightarrow\left(i_{n}, U_{n}\right)$.

It is now possible to explain how to decompose any element of $\tilde{e} \cdot\left(T_{S}(M) * G\right) \cdot \tilde{e}$ along the paths in $Q_{G}$ via the isomorphism $\mathbb{k} Q_{G} \stackrel{(1.1 .1)}{\longrightarrow} \tilde{e}\left(T_{S}(M) * G\right) \cdot \tilde{e}$. This is done with the following construction and assuming that $U=\mathbb{k}_{k} G_{i} \cdot \varepsilon_{U}$ for all vertices $(i, U)$ of $Q_{G}$. Given a tensor $m_{1} \otimes \cdots \otimes m_{n} * g \in e_{i_{0}} \cdot\left(M^{\otimes} n * G\right) \cdot e_{i_{n}}$, where $n \geqslant 0$ and $i_{0}, i_{n} \in\left[G \backslash Q_{0}\right]$, then

$m_{1} \otimes \cdots \otimes m_{n} * g \in\left({ }_{i_{0}} M_{y_{1} \cdot i_{1}} \otimes_{\mathrm{k}} \cdots \otimes_{\mathrm{k}}\left(y_{1} \cdots y_{n-1}\right) \cdot i_{n-1} M_{\left(y_{1} \cdots y_{n}\right) \cdot i_{n}}\right) * y_{1} \cdots y_{n} \mathbb{k} G_{i_{n}}$ for unique $i_{1}, \ldots, i_{n-1} \in\left[G \backslash Q_{0}\right]$ and $y_{t} \in\left[G / G_{i_{t}}\right]$, for all $t \in\{1, \ldots, n\}$; this defines an intertwiner, for all $U \in \operatorname{irr}\left(G_{i_{0}}\right)$ and $V \in \operatorname{irr}\left(G_{i_{n}}\right)$,

$$
\begin{array}{ccc}
\mathbb{k} G_{i_{0}} \cdot \varepsilon_{U} & \longrightarrow & M\left(i_{0}, \ldots, i_{n} ; \mathbb{k}_{k} G_{i_{n}} \cdot \varepsilon_{V}\right) \\
u \cdot \varepsilon_{U} & \longmapsto & \left(u * \varepsilon_{U}\right) \cdot\left(m_{1} \otimes \cdots \otimes m_{n} * g\right) \cdot\left(e_{i_{n}} * \varepsilon_{V}\right),
\end{array}
$$

where the products on the right-hand side are meant in $T_{S}(M) * G$; summing these intertwiners over all $U \in \operatorname{irr}\left(G_{i_{0}}\right)$ and $V \in \operatorname{irr}\left(G_{i_{n}}\right)$ defines an element of $\mathcal{I}$ ntw. This construction may be extended linearly to a linear mapping

$$
\hat{e} \cdot\left(T_{S}(M) * G\right) \cdot \hat{e} \longrightarrow \mathcal{I n t w}^{\text {op }},
$$

where $\hat{e}=\sum_{i \in\left[G \backslash Q_{0}\right]} e_{i}$. Denote by $\Xi$ the restriction of this mapping to $\tilde{e} \cdot\left(T_{S}(M) *\right.$ $G) \cdot \tilde{e}$,

$$
\Xi: \tilde{e} \cdot\left(T_{S}(M) * G\right) \cdot \tilde{e} \longrightarrow \text { Intw }^{\text {op }} .
$$

Remark 1.26. Here is how $\hat{e}$ and $\tilde{e}$ are related. Given idempotents $a$ and $b$ of an algebra $\Lambda$, say that $b$ is a reduction of $a$ whenever $\Lambda b$ is a direct summand of $\Lambda a$ in $\operatorname{Mod}(\Lambda)$ and the algebras $\operatorname{End}_{\Lambda}(\Lambda b)$ and $\operatorname{End}_{\Lambda}(\Lambda a)$ are Morita equivalent. Now $\tilde{e}$ $\left(=\sum_{i \in\left[G \backslash Q_{0}\right]} e_{i} *\left(\sum_{U \in \operatorname{irr}\left(G_{i}\right)} \varepsilon_{U}\right)\right)$ is a reduction of $\hat{e} * 1_{\mathrm{k} G}\left(=\sum_{i \in\left[G \backslash Q_{0}\right]} e_{i} * 1_{\mathrm{k} G_{i}}\right)$ in the algebra $T_{S}(M) * G$. 
Theorem 1.27. Let $Q_{0}, S, G$, and $M$ be as in Setting 1.1. Let $Q_{G}$ be as in Setting 1.4. Assume that $U=\mathbb{k} G_{i} \cdot \varepsilon_{U}$ for all vertices $(i, U)$ of $Q_{G}$. Then, $\Xi$, as introduced in (1.6.3), is an isomorphism of $\mathbb{k}$-algebras such that the following diagram is commutative,

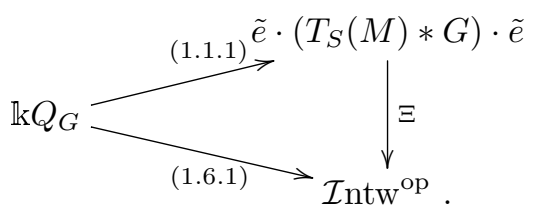

In particular, if (1.1.1) is used as an identification, then, for all $\theta \in \tilde{e} \cdot\left(T_{S}(M) * G\right) \cdot \tilde{e}$,

$$
\theta=\sum_{\gamma \text { path in } Q_{G}}\left(\Xi(\theta) \mid \varphi_{\gamma}\right) \cdot \gamma
$$

Example 1.28. This is the continuation of Example 1.24. Denote by $w$ the path $x_{\bullet}, x_{\overline{0}}, \overline{1} x_{\overline{1}, \bullet}$ in $Q$, this is an oriented cycle of length three going clockwise in the picture of $Q$ in Example 1.3. The present example describes the intertwiner $\Xi(\tilde{e}$. $(w * \mathrm{Id}) \cdot \tilde{e})$ as well as its preimage under the isomorphism $\mathbb{k}_{Q_{G}} \stackrel{(1.6 .1)}{\longrightarrow} \mathcal{I n t w}^{\mathrm{op}}$.

Because of the definition,

$$
\Xi(\tilde{e} \cdot(w * \mathrm{Id}) \cdot \tilde{e})=\sum_{s, t \in \mathbb{Z} / 2 \mathbb{Z}} \Xi(\tilde{e} \cdot(w * \mathrm{Id}) \cdot \tilde{e})_{s, t},
$$

where, for all $s, t \in \mathbb{Z} / 2 \mathbb{Z}$, the piece of notation $\Xi(\tilde{e} \cdot(w * \mathrm{Id}) \cdot \tilde{e})_{s, t}$ stands for the intertwiner $\rho_{s} \rightarrow M\left(\bullet, \overline{0}, \overline{0}, \bullet ; \rho_{t}\right)$ which maps $\varepsilon_{s}$ to $\left(e_{\bullet} \otimes \varepsilon_{s}\right) \cdot \tilde{e} \cdot(w \otimes \operatorname{Id}) \cdot \tilde{e} \cdot\left(e_{\bullet} \otimes \varepsilon_{t}\right)$. For all $s, t \in \mathbb{Z} / 2 \mathbb{Z}$, the latter expression is equal to $\left(1_{\mathbb{k} Q} \otimes \varepsilon_{s}\right) \cdot\left(w \otimes \varepsilon_{t}\right)$, which in turn is equal to $\frac{1}{2}\left(x_{\bullet, \overline{0}} x_{\overline{0}, \overline{1}} x_{\overline{1}, \bullet} \otimes \varepsilon_{t}+(-1)^{s+\overline{1}+t} x_{\bullet, \overline{1}} x_{\overline{1}, \overline{0}} x_{\overline{0}, \bullet} \otimes \varepsilon_{t}\right)$; therefore, using the notation of Example 1.11,

$$
\Xi(\tilde{e} \cdot(w * \mathrm{Id}) \cdot \tilde{e})_{s, t}=F_{\overline{0}} .
$$

Now, let $s, t, a \in \mathbb{Z} / 2 \mathbb{Z}$; using the computation of the pairings of the shape $\left(F_{a} \mid \phi_{b}\right)$ made in Example 1.21 as well as the description of the intertwiners relative to $M^{*}$ associated to paths in $Q_{G}$ made in Example 1.24, and denoting by $\gamma$ the path $\left(\bullet, \rho_{s}\right) \stackrel{b_{s}}{\longrightarrow}\left(\overline{0}, \mathbb{k}_{\mathrm{k}} \cdot \mathrm{Id}\right) \stackrel{d_{a}}{\longrightarrow}\left(\overline{0}, \mathbb{k}_{k} \cdot \mathrm{Id}\right) \stackrel{c_{t}}{\longrightarrow}\left(\bullet, \rho_{t}\right)$ of $Q_{G}$,

$$
\left(\Xi(\tilde{e}(w \otimes \mathrm{Id}) \cdot \tilde{e})_{s, t} \mid \varphi_{\gamma}\right)= \begin{cases}\frac{1+(-1)^{t}}{2} & \text { if } a=\overline{0} \\ 0 & \text { if not; }\end{cases}
$$

Therefore $\Xi(\tilde{e} \cdot(w \otimes \mathrm{Id}) \cdot \tilde{e})_{s, t}=\frac{1+(-1)^{t}}{2} \cdot b_{s} d_{\overline{0}} c_{t}$ (see (1.6.5)). As a whole, $\Xi(\tilde{e}$. $(w \otimes \mathrm{Id}) \cdot \tilde{e})=b_{\overline{0}} d_{\overline{0}} c_{\overline{0}}+b_{\overline{1}} d_{\overline{0}} c_{\overline{0}}$.

\section{Practical aspects}

Let $Q_{0}, S, G$ and $M$ be as in Setting 1.1.

Applying Theorem 1.27 for computations requires to compute $Q_{G}$ first. Actually, rather than computing explicitly the intertwiners relative to $M$ which form the arrows of $Q_{G}$, it is simpler to

(1) compute a basis of $\operatorname{Hom}_{\mathbb{k} G_{j}}\left(\tau, M^{*}(j, i ; \rho)\right)$ for all pairs $(i, \rho)$ and $(j, \tau)$ such that $i, j \in\left[G \backslash Q_{0}\right], \rho \in \operatorname{irr}\left(G_{i}\right)$, and $\tau \in \operatorname{irr}\left(G_{j}\right)$.

Because of the pairing $(-\mid-)$, this yields a quiver $Q_{G}$ such as in Setting 1.4 as well as the intertwiners $\varphi_{\alpha}$ for all arrows $\alpha$ of $Q_{G}$. This avoids computing explicitly the intertwiners corresponding to the arrows of $Q_{G}$, which are not used in the formulas of Theorem 1.27. 
Given an element $\theta$ of $\tilde{e} \cdot\left(T_{S}(M) * G\right) \cdot \tilde{e}$, say homogeneous of degree $n$ for the grading induced by the tensor powers of $M$, the decomposition into a linear combination of paths in $Q_{G}$ of the inverse image of $\theta$ under the isomorphism $\mathbb{k} Q_{G} \stackrel{(1.1 .1)}{\longrightarrow} \tilde{e} \cdot\left(T_{S}(M) * G\right) \cdot \tilde{e}$ may be computed as follows using Theorem 1.27,

(2) compute $\Xi(\theta)$, which is a sum of intertwiners relative to $M$, see (1.6.3);

(3) compute the intertwiners $\varphi_{\gamma}$ for all paths $\gamma$ of length $n$ in $Q_{G}$, see Notation 1.23

(4) compute the pairing $\left(\Xi(\theta) \mid \varphi_{\gamma}\right)$ for all such paths $\gamma$, see Proposition 1.20.

The desired linear combination of paths is hence $\sum_{\gamma}\left(\Xi(\theta) \mid \varphi_{\gamma}\right) \cdot \gamma$ (see (1.6.5)).

These computations may be performed by a computer that is able to determine the irreducible representations of $\operatorname{irr}\left(G_{i}\right)$ for all $i \in\left[G \backslash Q_{0}\right]$. This section illustrates these computations.

2.1. How to pair intertwiners? In view of (1.4.2), the main difficulty in the computations listed in the introduction of Section 2 lies in computing pairings for $(-\mid-)$. This section explains how to compute (1.5.2) in combinatorial terms in the following specific setting.

Setting 2.1. Let $Q_{0}, S, G$ and $M$ be as in Setting 1.1. Let $Q$ be a quiver with vertex set being $Q_{0}$ and such that $M$ is equal to the vector space with basis elements being the arrows of $Q$. Let $Q_{G}$ be as in Setting 1.4. Assume that

- the stabiliser $G_{i}$ is abelian for all $i \in Q_{0}$,

- $U=\mathbb{k}_{k} G_{i} \cdot \varepsilon_{U}$ for all vertices $(i, U)$ of $Q_{G}$, denote by $\chi_{U}$ the character $G_{i} \rightarrow \mathbb{k}^{\times}$of $U$,

- and, for all $g \in G$ and all paths $\gamma$ in $Q$, there exists a scalar $\chi_{g, \gamma} \in \mathbb{k}^{\times}$and a path $g(\gamma)$ such that ${ }^{g} \gamma=\chi_{g, \gamma} g(\gamma)$.

If $G$ is abelian, then it is possible to find a quiver $Q$ and to choose irreducible representations $U$ of $G_{i}$, for all $i \in\left[G \backslash Q_{0}\right]$, fitting in this setting.

Notation 2.2. The following notation relative to $M$ is useful.

- For all arrows $a: i \rightarrow j$ of $Q$, denote by $a^{*}$ the element of $M^{*}$ such that $a^{*}(a)=1$ and $a^{*}(b)=0$ for all arrows $b$ of $Q$ distinct from $a$.

- Denote by $Q^{*}$ the quiver with vertex set being $Q_{0}$ and whose arrows are the linear forms $a^{*}$ introduced just before. This quiver is isomorphic to the opposite quiver of $Q$.

- For all paths $\gamma: t_{0} \stackrel{a_{1}}{\longrightarrow} t_{1} \rightarrow \cdots \rightarrow t_{n-1} \stackrel{a_{n}}{\longrightarrow} t_{n}$ in $Q$, denote by $\gamma^{*}$ the path $t_{n} \stackrel{a_{n}^{*}}{\longrightarrow} t_{n-1} \rightarrow \cdots \rightarrow t_{1} \stackrel{a_{1}^{*}}{\longrightarrow} t_{0}$ in $Q^{*}$.

Note that every path in $Q$ starting in some vertex $i_{0}$ lying in $\left[G \backslash Q_{0}\right]$ is of the shape $i_{0} \rightarrow y_{1} \cdot i_{1} \rightarrow \cdots \rightarrow\left(y_{1} \cdots y_{n}\right) \cdot i_{n}$ for a unique sequence $i_{1}, \ldots, i_{n}$ in $\left[G \backslash Q_{0}\right]$ and a unique sequence $y_{1}, \ldots, y_{n}$ in $G$ such that $y_{t} \in\left[G / G_{i_{t}}\right]$ for all $t \in\{1, \ldots, n\}$.

Let $\underline{i}=i_{0}, \ldots, i_{n}$ be a sequence in $\left[G \backslash Q_{0}\right]$, consider $U \in \operatorname{irr}\left(G_{i_{0}}\right)$ and $V \in$ $\operatorname{irr}\left(G_{i_{n}}\right)$, and let $f: U \rightarrow M(\underline{i} ; V)$ and $\varphi: V \rightarrow M^{*}\left(\underline{i}^{o} ; U\right)$ be intertwiners. Since the representation $V$ is one dimensional, then,

$$
f\left(\varepsilon_{u}\right)=\sum_{\gamma} \alpha_{\gamma} \gamma \otimes y_{1} y_{2} \cdots y_{n} \varepsilon_{V}
$$

where $\gamma$ runs through all paths in $Q$ of the shape

$$
i_{0} \rightarrow y_{1} \cdot i_{1} \rightarrow y_{1} y_{2} \cdot i_{2} \rightarrow \cdots \rightarrow\left(y_{1} \cdots y_{n}\right) \cdot i_{n}
$$

for some (unique) sequence $y=y_{1}, \ldots, y_{n}$ such that $y_{t} \in\left[G / G_{i_{t}}\right]$ for all $t \in$ $\{1, \ldots, n\}$, and $\alpha_{\gamma} \in \mathbb{k}$. Note that the considered paths are precisely the ones of the 
shape $i_{0} \rightarrow j_{1} \rightarrow \cdots \rightarrow j_{n}$ such that $j_{t}$ lies in the orbit of $i_{t}$ for all $t \in\{1, \ldots, n\}$. Similarly,

$$
\varphi\left(\varepsilon_{V}\right)=\sum_{\gamma^{\prime}} \beta_{\gamma^{\prime}} \gamma^{\prime} \otimes x_{n-1} \cdots x_{0} \varepsilon_{U},
$$

where $\gamma^{\prime}$ runs through all paths in $Q$ of the shape

$$
i_{n} \rightarrow x_{n-1} \cdot i_{n-1} \rightarrow x_{n-1} x_{n-2} \cdot i_{n-2} \rightarrow \cdots \rightarrow\left(x_{n-1} x_{n-2} \cdots x_{0}\right) \cdot i_{0}
$$

for some (unique) sequence $\underline{x}=x_{n-1}, \ldots, x_{0}$ such that $x_{t} \in\left[G / G_{i_{t}}\right]$ for all $t \in$ $\{0, \ldots, n-1\}$, and $\beta_{\gamma^{\prime}} \in \mathbb{k}$.

Lemma 2.3. Using the decompositions (2.1.1) and (2.1.2), then

$$
(f \mid \varphi)=\sum_{\gamma} \alpha_{\gamma} \cdot \beta_{\left(y_{1} \cdots y_{n}\right)^{-1}(\gamma)^{*}} \cdot \chi_{\left(y_{1} \cdots y_{n}\right)^{-1}, \gamma} \cdot \chi_{U}\left(h_{0}\right),
$$

where $\gamma$ runs through all paths in $Q$ of the shape $i_{0} \rightarrow j_{1} \rightarrow \cdots \rightarrow j_{n}$ such that $j_{t}$ lies in the orbit of $i_{t}$ for all $t \in\{1, \ldots, n\}$, where $\alpha_{\gamma} \in \mathbb{k}$, and where, for all such paths $\gamma$,

- $\underline{y}=y_{1}, \ldots, y_{n}$ denotes the sequence in $G$ such that $y_{t} \in\left[G / G_{i_{t}}\right]$, for all $\bar{t} \in\{1, \ldots, n\}$ and $\gamma$ is a path of the shape $i_{0} \rightarrow y_{1} \cdot i_{1} \rightarrow \cdots \rightarrow y_{1} \cdots y_{n} \cdot i_{n}$,

- $h_{0}$ is the element of $G_{i_{0}}$ such as in (c) in Proposition 1.20,

- $\chi\left(y_{1} \cdots y_{n}\right)^{-1}, \gamma$ is the element of $\mathbb{k}^{\times}$and $\left(y_{1} \cdots y_{n}\right)^{-1}(\gamma)$ is the path in $Q$ such that $\left(y_{1} \cdots y_{n}\right)^{-1} \gamma=\chi_{\left(y_{1} \cdots y_{n}\right)^{-1}, \gamma}\left(y_{1} \cdots y_{n}\right)^{-1}(\gamma)$,

- and $\left(\left(y_{1} \cdots y_{n}\right)^{-1}(\gamma)\right)^{*}$ is the reverse path in $Q^{*}$ associated to the path $\left(y_{1} \cdots y_{n}\right)^{-1}(\gamma)$ in $Q$.

Proof. For all sequences $y=y_{1}, \ldots, y_{n}$ and $\underline{x}=x_{n-1}, \ldots, x_{0}$ such that $x_{t} \in\left[G / G_{i_{t}}\right]$ and $y_{t} \in\left[G / G_{i_{t}}\right]$ for all $\bar{t}$, denote by $\mathcal{C}(\underline{y})$ and $\mathcal{C}^{\prime}(\underline{x})$ the sets of paths in $Q$ and in $Q^{*}$ of the shape $i_{0} \rightarrow y_{1} \cdot i_{1} \rightarrow \cdots \rightarrow y_{1} \cdots y_{n} \cdot i_{n}$ and $i_{n} \rightarrow x_{n-1} \cdot i_{n-1} \rightarrow \cdots \rightarrow$ $x_{n-1} \cdots x_{0} \cdot i_{0}$, respectively. For all $y$,

$$
f_{\underline{y}}\left(\varepsilon_{U}\right)=\sum_{\gamma \in \mathcal{C}(\underline{y})} \alpha_{\gamma} \gamma \otimes y_{1} \cdots y_{n} \varepsilon_{U}
$$

hence, the following term that serves in the definition of $(f \mid \varphi)$, see (1.5.2),

$$
\left(y_{1} \cdots y_{n}\right)^{-1} f_{\underline{y}}^{(1)}\left(\varepsilon_{U}\right) \otimes \cdots \otimes{ }^{\left(y_{1} \cdots y_{n}\right)^{-1}} f_{\underline{y}}^{(n)}\left(\varepsilon_{U}\right) \otimes f_{\underline{y}}^{(0)}\left(\varepsilon_{U}\right),
$$

is equal to

$$
\sum_{\gamma \in \mathcal{C}(\underline{y})} \alpha_{\gamma} \cdot \chi_{\left(y_{1} \cdots y_{n}\right)^{-1}, \gamma} \cdot \underbrace{\left(y_{1} \cdots y_{n}\right)^{-1}(\gamma)}_{\in M^{\otimes} S^{n}} \otimes \varepsilon_{U} .
$$

Similarly, for all $\underline{x}$,

$$
\varphi^{\underline{x}}\left(\varepsilon_{V}\right)=\sum_{\gamma^{\prime} \in \mathcal{C}(\underline{x})} \beta_{\gamma^{\prime}} \gamma^{\prime} \otimes x_{n-1} \cdots x_{0} \varepsilon_{U} .
$$

Therefore, given $\underline{y}$, the " $\Pi_{t}$ "-term in (1.5.2) is equal to

$$
\sum_{\gamma \in \mathcal{C}(\underline{y}), \gamma^{\prime} \in \mathcal{C}(\underline{x})} \alpha_{\gamma} \cdot \beta_{\gamma^{\prime}} \chi_{\left(y_{1} \cdots y_{n}\right)^{-1}, \gamma} \cdot b_{n}^{*}\left(c_{n}\right) \cdots b_{2}^{*}\left(c_{2}\right) \cdot b_{1}^{*}\left(c_{1}\right) \cdot \chi_{U}\left(h_{0}\right) \cdot \varepsilon_{U},
$$

where

- $\underline{x}=x_{n-1}, \ldots, x_{0}$ and $h_{0}$ are the elements of $G$ determined by $\underline{y}$ and by (a), (b), and (c) in Proposition 1.20,

- $b_{1}^{*}, \ldots, b_{n}^{*}$ are the arrows of $Q^{*}$ such that $\gamma^{\prime}$ is $\stackrel{b_{n}^{*}}{\longrightarrow} \cdot \stackrel{b_{n-1}^{*}}{\longrightarrow} \cdots \stackrel{b_{1}^{*}}{\longrightarrow} \cdot$

- and $c_{1}, \ldots, c_{n}$ are the arrows of $Q$ such that $\left(y_{1} \cdots y_{n}\right)^{-1}(\gamma)$ is $\stackrel{c_{1}}{\longrightarrow} \cdot \stackrel{c_{2}}{\longrightarrow}$ ... $\stackrel{c_{n}}{\longrightarrow} \cdot$ 
By definition of the arrows $b_{1}^{*}, \ldots, b_{n}^{*}$, the summand of (2.1.4) with index $\gamma$ is nonzero only if the paths $\cdot \stackrel{b_{1}}{\longrightarrow} \cdot \stackrel{b_{2}}{\longrightarrow} \cdots \stackrel{b_{n}}{\longrightarrow} \cdot$ and $\left(y_{1} \cdots y_{n}\right)^{-1}(\gamma)$ in $Q$ are equal, that is, only if $\gamma^{\prime}=\left(\left(y_{1} \cdots y_{n}\right)^{-1}(\gamma)\right)^{*}$. Summing (2.1.4) over all possible $y$ yields that $(f \mid \varphi) \varepsilon_{U}$ is equal to

$$
\sum_{\gamma} \alpha_{\gamma} \beta_{\left(\left(y_{1} \cdots y_{n}\right)^{-1}(\gamma)\right)^{*}} \chi_{\left(y_{1} \cdots y_{n}\right)^{-1}, \gamma} \chi_{U}\left(h_{0}\right) \varepsilon_{U}
$$

This proves (2.1.3).

2.2. An example. In this example, $Q$ is the following quiver whose vertices are the elements of $\mathbb{Z} / 4 \mathbb{Z}$

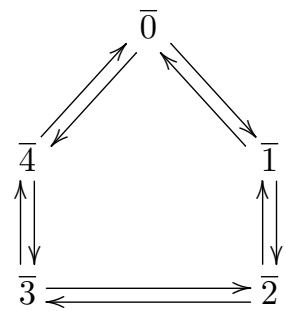

and $G$ is the dihedral group of order 10

$$
G=\left\langle c, \tau \mid c^{5}, \tau^{2}, \tau c \tau c\right\rangle .
$$

Denote by $\varepsilon$ the group homomorphism $G \rightarrow\{-1,1\}$ such that $\varepsilon(c)=1$ and $\varepsilon(\tau)=$ -1 . Denote the arrows of $Q$ by $x_{i, j}: i \rightarrow j$. For convenience, denote the arrows of $Q^{*}$ by $x_{i, j}^{\prime}: i \rightarrow j$, hence $\left(x_{j, i}\right)^{*}=x_{i, j}^{\prime}$. The actions of $G$ on $Q_{0}$ and $M$ are assumed to be the ones such that, for all $i \in Q_{0}$ and $g \in G$, and for all arrows $x_{i, j}$ of $Q$,

$$
c \cdot i=i+\overline{1}, \tau \cdot i=-i,{ }^{g} x_{i, j}=\varepsilon(g) x_{g \cdot i, g \cdot j} .
$$

The purpose of this section is to make a full illustration of Theorem 1.27, that is, for all $w \in T_{S}(M) * G$,

- to describe the intertwiner $\Xi(\theta)$, where $\theta$ denotes $\tilde{e} \cdot w \cdot \tilde{e}$;

- and to describe the preimage of $\theta$ under the isomorphism $\mathbb{k}_{k} Q_{G} \stackrel{(1.1 .1)}{\longrightarrow}$ $\tilde{e} \cdot\left(T_{S}(M) * G\right) \cdot \tilde{e}$.

Let $\left[G \backslash Q_{0}\right]$ be $\{\overline{0}\}$. Note that $G_{\overline{0}}=\{\operatorname{Id}, \tau\}$. Let $\left[G / G_{\overline{0}}\right]$ be $\left\{\operatorname{Id}, c, c^{2}, c^{3}, c^{4}\right\}$. For all $s \in\{0,1\}$, denote by $\varepsilon_{s}$ the following primitive idempotent of $\mathbb{k}_{k} G_{\overline{0}}$,

$$
\varepsilon_{s}=\frac{1}{2}\left(\mathrm{Id}+(-1)^{s} \tau\right)
$$

and denote $\varepsilon_{s} \cdot \mathbb{k}_{k} G_{\overline{0}}$ by $\rho_{s}$. Finally, let $\operatorname{irr}\left(G_{\overline{0}}\right)$ be equal to $\left\{\rho_{0}, \rho_{1}\right\}$. In particular,

$$
\tilde{e}=e_{\overline{0}} * \varepsilon_{0}+e_{\overline{0}} * \varepsilon_{1} .
$$

The setting presented below is assumed until the end of the subsection. In view of the purpose stated previously, this is not a loss of generality in making this assumption because

- the elements of the shape $p * g$, where $p$ is a path in $Q$ and $g \in G$, form a basis of $T_{S}(M) * G$ as a vector space;

- every $g \in G$ is equal to $c^{\ell} \tau^{r}$ for a unique couple $(\ell, r) \in\{0,1,2,3,4\} \times\{0,1\}$;

- for all paths $p$ of $Q$ and for all $(\ell, r) \in\{0,1,2,3,4\} \times\{0,1\}$, the product $\tilde{e} \cdot(p * g) \cdot \tilde{e}$ vanishes as soon as $p$ is not a path from $\overline{0}$ to $-\bar{\ell}$.

Setting 2.4. Assume that $w$ is equal to $p * c^{\ell} \tau^{r}$, where $(\ell, r) \in\{0,1,2,3,4\} \times\{0,1\}$ and $p$ is a path in $Q$ from $\overline{0}$ to $-\bar{\ell}$. Denote the length of $p$ by $n$. Denote by $i_{0}, \ldots, i_{n}$ the sequence of vertices of $Q$ such that $p$ has the shape $i_{0} \rightarrow i_{1} \rightarrow \cdots \rightarrow i_{n}$. Thus, 

$p=x_{i_{0}, i_{1}} x_{i_{1}, i_{2}} \cdots x_{i_{n-1}, i_{n}}$. In particular, $i_{0}=\overline{0}, i_{n}=-\bar{\ell}$ and $i_{k}-i_{k-1} \in\{-\overline{1}, \overline{1}\}$ for all $k \in\{1, \ldots, n\}$.

2.2.1. Computation of $Q_{G}$. By definition, for all $t \in\{0,1\}$,

$$
M^{*}\left(\overline{0}, \overline{0} ; \rho_{t}\right)=\operatorname{Span}\left(x_{\overline{0}, \overline{1}}^{\prime} \otimes c \varepsilon_{t}, x_{\overline{0}, \overline{4}}^{\prime} \otimes c^{4} \varepsilon_{t}\right),
$$

where the action of $\tau$ is given as follows

$$
\left\{\begin{aligned}
x_{\overline{0}, \overline{1}}^{\prime} \otimes c \varepsilon_{t} & \longmapsto(-1)^{1+t} x_{\overline{0}, \overline{4}}^{\prime} \otimes c^{4} \varepsilon_{t} \\
x_{\overline{0}, \overline{4}}^{\prime} \otimes c^{4} \varepsilon_{t} & \longmapsto(-1)^{1+t} x_{\overline{0}, \overline{1}}^{\prime} \otimes c \varepsilon_{t} .
\end{aligned}\right.
$$

Hence, for all $s, t \in\{0,1\}$,

$$
\operatorname{Hom}_{\mathrm{k} G_{\overline{0}}}\left(\rho_{s}, M^{*}\left(\overline{0}, \overline{0} ; \rho_{t}\right)\right)=\operatorname{Span}\left(\varphi_{s, t}\right),
$$

where $\varphi_{s, t}$ is the intertwiner such that $\varphi_{s, t}\left(\varepsilon_{s}\right)=\varepsilon_{s} \cdot\left(x_{\overline{0}, \overline{1}}^{\prime} \otimes c \varepsilon_{t}\right)\left(=\varepsilon_{s} \cdot\left(x_{\overline{0}, \overline{1}}^{\prime} \otimes c\right) \cdot \varepsilon_{t}\right)$, that is,

$$
\varphi_{s, t}\left(\varepsilon_{s}\right)=x_{\overline{0}, \overline{1}}^{\prime} \otimes c \varepsilon_{t}+(-1)^{s+t+1} x_{\overline{0}, \overline{4}}^{\prime} \otimes c^{4} \varepsilon_{t} .
$$

Denote by $f_{s, t}$ the intertwiner $\rho_{s} \rightarrow M\left(\overline{0}, \overline{0} ; \rho_{t}\right)$ such that $\left(f_{s, t} \mid \varphi_{t, s}\right)=1$, for all $s, t \in\{0,1\}$. Then $Q_{G}$ may be taken equal to the following quiver

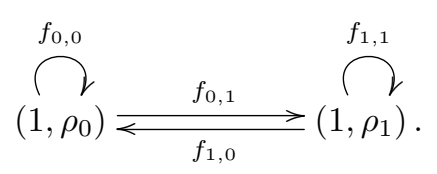

2.2.2. The intertwiner $\Xi(\theta)$. By definition, $\Xi(\theta)$ decomposes as

$$
\Xi(\theta)=\sum_{s, t \in\{0,1\}} \Xi(\theta)_{s, t},
$$

where, for all $s, t \in\{0,1\}$, the intertwiner $\Xi(\theta)_{s, t}: \rho_{s} \rightarrow M(\underbrace{0, \ldots, 0}_{n+1} ; \rho_{t})$ is such that $\Xi(\theta)_{s, t}\left(\varepsilon_{s}\right)=\varepsilon_{s} \cdot \tilde{e} \cdot w \cdot \tilde{e} \cdot \varepsilon_{t}$, see (1.6.3). Note that

$$
\begin{aligned}
\Xi(\theta)_{s, t}\left(\varepsilon_{s}\right) & =\varepsilon_{s} \cdot \tilde{e} \cdot w \cdot \tilde{e} \cdot \varepsilon_{t} \\
& =\frac{1}{2}\left(\operatorname{Id}+(-1)^{s} \tau\right) \cdot\left(p \otimes c^{\ell} \tau^{r}\right) \cdot \varepsilon_{t} \\
& =\frac{(-1)^{r t}}{2}\left(\operatorname{Id}+(-1)^{s} \tau\right) \cdot\left(p \otimes c^{\ell} \varepsilon_{t}\right) \\
& =\frac{(-1)^{r t}}{2}\left(p \otimes c^{\ell} \varepsilon_{t}+(-1)^{s+t}\left({ }^{\tau} p\right) \otimes c^{-\ell} \varepsilon_{t}\right)
\end{aligned}
$$

where ${ }^{\tau} p=(-1)^{n} x_{-i_{0},-i_{1}} x_{-i_{1},-i_{2}} \cdots x_{-i_{n-1},-i_{n}}$.

2.2.3. Intertwiners relative to $M^{*}$ associated to paths of $Q_{G}$. Consider a path of length denoted by $m$ in $Q_{G}$, say $q:\left(0, \rho_{s_{0}}\right) \rightarrow\left(0, \rho_{s_{1}}\right) \rightarrow \cdots \rightarrow\left(0, \rho_{s_{m}}\right)$, where $s_{0}, \ldots, s_{m} \in\{-1,1\}$. Then $\varphi_{q}$ is the following intertwiner $\rho_{s_{m}} \rightarrow M^{*}(\underbrace{0, \ldots, 0}_{n+1} ; \rho_{s_{0}})$,

$$
\varphi_{q}=\varphi_{s_{1}, s_{0}} \circledast \varphi_{s_{2}, s_{1}} \circledast \cdots \circledast \varphi_{s_{m}, s_{m-1}} .
$$

Consider $\varphi_{q}\left(\varepsilon_{s_{m}}\right)$ as an element of $\mathbb{k} Q^{*} * G$; following (1.4.2) it equals the product $\left(x_{\overline{0}, \overline{1}}^{\prime} \otimes(-1)^{s_{m}+s_{m-1}+1} x_{\overline{0}, \overline{4}} \otimes c^{4}\right) \cdots\left(x_{\overline{0}, \overline{1}}^{\prime} \otimes(-1)^{s_{1}+s_{0}+1} x_{\overline{0}, \overline{4}} \otimes c^{4}\right)$. Hence

$$
\varphi_{q}\left(\varepsilon_{s_{m}}\right)=\sum(-1)^{\sum s_{k}+s_{k-1}+1} x_{\overline{0}, j_{m-1}}^{\prime} x_{j_{m-1} j_{m-2}}^{\prime} \cdots x_{j_{1}, j_{0}}^{\prime} \otimes c^{j_{0}},
$$

where

- the first sum runs over all sequences $j_{m-1}, \ldots, j_{0} \in \mathbb{Z} / 4 \mathbb{Z}$ such that the expression $x_{\overline{0}, j_{m-1}}^{\prime} \cdots x_{j_{1}, j_{0}}^{\prime}$ is a path in $Q^{\prime}$, which means that $j_{t}-j_{t-1} \in$ $\{-\overline{1}, \overline{1}\}$ for all $t \in\{1, \ldots, m\}$ (taking $j_{m}=\overline{0}$ ), 
- and the second sum (in the exponent) runs over all indices $k \in\{1, \ldots, m\}$ such that $x_{j_{k}, j_{k-1}}^{\prime}$ of $Q^{\prime}$ goes anticlockwise when $Q^{\prime}$ is drawn like $Q$ in (2.2.1), which means that $j_{k}-j_{k-1}=\overline{1}$.

2.2.4. Computation of the pairing $\left(\Xi(\theta) \mid \varphi_{q}\right)$. Let $q:\left(0, \rho_{s_{0}}\right) \rightarrow\left(0, \rho_{s_{1}}\right) \rightarrow \cdots \rightarrow$ $\left(0, \rho_{s_{m}}\right)$ be a path in $Q_{G}$ like in 2.2.3. Because of the definition of the action of $G$ on $\mathbb{k} Q$, the pairing $\left(\Xi(\theta) \mid \varphi_{q}\right)\left(=\left(\Xi(\theta)_{s_{0}, s_{m}} \mid \varphi_{q}\right)\right)$ is given by (2.1.3). In particular, because of (2.2.3) and (2.2.4),

- this pairing vanishes if $m \neq n$, that is, $p$ and $q$ have different lengths,

- if $m=n$ then, for all paths $\gamma$ distinct from both $x_{i_{0}, i_{1}} x_{i_{1}, i_{2}} \cdots x_{i_{n-1}, i_{n}}$ and $x_{i_{0},-i_{1}} x_{-i_{1},-i_{2}} \cdots x_{-i_{n-1},-i_{n}}$, the term of index $\gamma$ in (2.2.3) vanishes.

Using (2.2.3) and (2.2.4), the scalars appearing in (2.1.3) may be computed directly for these two possibilities for $\gamma$, assuming that $m=n$. Table 7 describes these scalars, they appear in bold face. Recall that $i_{0}=\overline{0}$.

\begin{tabular}{|c|c|c|}
\hline$\gamma$ & $x_{i_{0}, i_{1}} x_{i_{1}, i_{2}} \cdots x_{i_{n-1}, i_{n}}$ & $x_{i_{0},-i_{1}} x_{-i_{1},-i_{2}} \cdots x_{-i_{n-1},-i_{n}}$ \\
\hline$\alpha_{\gamma}$ & $\frac{(-1)^{r s_{n}}}{2}$ & $(-1)^{r s_{n}+s_{0}+s_{n}+\mathbf{n}}$ \\
\hline$y_{1}, \ldots, y_{n}$ & $c^{i_{1}-i_{0}}, c^{i_{2}-i_{1}}, \ldots, c^{i_{n}-i_{n-1}}$ & $c^{-i_{1}+i_{0}}, c^{-i_{2}+i_{1}^{2}}, \ldots, c^{-i_{n}}{ }_{n}+i_{n-1}$ \\
\hline $\begin{array}{l}y_{1} \cdots y_{n} \\
\left(y_{1} \cdots y_{n}\right)^{-1} \gamma\end{array}$ & $x_{-i_{n}, i_{1}-i_{n} x_{i_{1}-i_{n}, i_{2}-i_{n}} \cdots x_{i_{n-1}-i_{n}, \overline{0}}}$ & $\begin{array}{c}c^{-i_{n}} \\
x_{i_{n}, i_{n}-i_{1}} x_{i_{n}-i_{1}, i_{n}-i_{2}} \cdots x_{i_{n}-i_{n-1}, \overline{0}}\end{array}$ \\
\hline $\begin{array}{l}\chi_{\left(\mathbf{y}_{1} \cdots \mathbf{y}_{\mathbf{n}}\right)^{-1}, \gamma}, \gamma \\
\left(y_{1} \cdots y_{n}\right)^{-1}(\gamma)^{*}\end{array}$ & $x_{\overline{0}, i_{n-1}-i_{n}}^{\prime} \cdots x_{i_{2}-i_{n}, i_{1}-i_{n}}^{\prime} x_{i_{1}-i_{n},-i_{n}}^{\prime}$ & $x_{\overline{0}, i_{n}-i_{n-1}}^{\prime} \cdots x_{i_{n}-i_{2}, i_{n}-i_{1}}^{\prime} x_{i_{n}-i_{1}, i_{n}}^{\prime}$ \\
\hline$\beta\left(\mathbf{y}_{\mathbf{1}} \cdots \mathbf{y}_{\mathbf{n}}\right)^{-1}(\gamma)^{*}$ & $\begin{array}{l}(-\mathbf{1})^{\mathbf{x}} \text { where } x \text { is } \\
\sum_{k \leqslant n} \quad s_{k}+s_{k-1}+1 \\
\text { s.t. } i_{k}-i_{k-1}=\overline{1}\end{array}$ & $\begin{array}{ll} & (-\mathbf{1})^{\mathbf{x}}, \text { where } x \text { is } \\
& \sum_{k \leqslant n} \quad s_{k}+s_{k-1}+1 \\
\text { s.t. } i_{k}- & i_{k-1}=-\overline{1}\end{array}$ \\
\hline $\begin{array}{l}h_{0} \\
\chi_{\rho_{\mathbf{S}_{\mathbf{0}}}}\left(\mathbf{h}_{\mathbf{0}}\right)\end{array}$ & $\begin{array}{c}\text { Id } \\
1\end{array}$ & $\begin{array}{c}\text { Id } \\
1\end{array}$ \\
\hline
\end{tabular}

TABLE 7. The coefficients in the expression (2.1.3) of $\left(\Xi(\theta) s_{0}, s_{m} \mid \varphi_{q}\right)$ according to $\gamma$

Thus

$$
\begin{aligned}
\left(\Xi(\theta) \mid \varphi_{q}\right)= & \frac{(-1)^{r s_{0}}}{2}(-1)^{\sum_{i_{k}-i_{k-1}=\overline{1}}\left(s_{k}+s_{k-1}+1\right)} \\
& +\frac{(-1)^{r s_{0}+s_{0}+s_{n}+n}}{2}(-1)^{\sum_{i_{k}-i_{k-1}=-\overline{1}}\left(s_{k}+s_{k-1}+1\right)} .
\end{aligned}
$$

As whole (after factoring and simplifying),

$$
\left(\Xi(\theta) \mid \varphi_{q}\right)= \begin{cases}(-1)^{r s_{0}}(-1)^{\sum_{i_{k}-i_{k-1}=\overline{1}}\left(s_{k}+s_{k-1}+1\right)} & \text { if } m=n \\ 0 & \text { otherwise. }\end{cases}
$$

2.2.5. Decomposition of $\theta$ into paths in $Q_{G}$. Because of Theorem 1.27, the preimage of $\theta$ under the isomorphism $\mathbb{k}_{k} Q_{G} \stackrel{(1.1 .1)}{\longrightarrow} \tilde{e} \cdot\left(T_{S}(M) * G\right) \cdot \tilde{e}$ is equal to

$$
\sum_{q \text { path in } Q_{G}}\left(\Xi(\theta) \mid \varphi_{q}\right) \cdot q \text {. }
$$

In view of (2.2.5), this preimage is hence equal to

$$
\sum_{s_{0}, \ldots, s_{n} \in\{0,1\}}(-1)^{r s_{0}}(-1)^{\sum_{i_{k}-i_{k-1}=\overline{1}} s_{k}+s_{k-1}+1} \cdot\left(\left(0, \rho_{s_{0}}\right) \rightarrow\left(0, \rho_{s_{1}}\right) \rightarrow \cdots \rightarrow\left(0, \rho_{s_{n}}\right)\right) .
$$

2.2.6. Illustration. Assume that $w=x_{\overline{0}, \overline{1}} x_{\overline{1}, \overline{2}} x_{\overline{2}, \overline{3}} x_{\overline{3}, \overline{4}} x_{\overline{4}, \overline{0}} * \operatorname{Id}-x_{\overline{0}, \overline{4}} x_{\overline{4}, \overline{3}} x_{\overline{3}, \overline{2}} x_{\overline{2}, \overline{1}} x_{\overline{1}, \overline{0}} *$ Id. Applying 2.2.5 yields that the preimage of $\tilde{e} \cdot w \cdot \tilde{e}$ under the isomorphism $\mathbb{k} Q_{G} \stackrel{(1.1 .1)}{\longrightarrow} \tilde{e} \cdot\left(T_{S}(M) * G\right) \cdot \tilde{e}$ is equal to $-2 \sum_{\gamma} \gamma$, where $\gamma$ runs through all oriented cycles of length 5 in $Q_{G}$. 


\section{Details on the monoidal CATEgory of Bimodules over Groups}

This section details some isomorphisms and properties in the monoidal category $\left(\bmod \left(A^{e}\right), \otimes_{A}\right)$ and related to the operation, say $\times$, and the pairing $\langle-\mid-\rangle$ considered in Remark 1.7.

- Section 3.1 describes the left dual of a tensor product of objects in $\bmod \left(A^{e}\right)$.

- Section 3.2 details the the adjunction $\left(X \otimes_{A}-\right) \vdash\left(X^{*} \otimes_{A}-\right)$ for a given $X \in \bmod \left(A^{e}\right)$.

- Section 3.3 details the compatibility of $\langle-\mid-\rangle$.

The existence of the isomorphisms and adjunctions discussed in these sections may be part of the folklore (see [5, Exercise 2.10.16]). However, the proof of Theorem 1.25 is based on their description, which is the reason for detailing these here. For the sake of simplicity, the presentation is made independently of the monoidal category $\left(\bmod \left(A^{e}\right), \otimes_{A}\right)$ and of the framework of Theorem 1.25.

Here are some conventions. In any group, $e$ denotes the neutral element; and Hom $_{\mathbb{k} G}$ and $\otimes_{\mathbb{k} G}$ are denoted by $\operatorname{Hom}_{G}$ and $\otimes_{G}$, respectively, for all finite groups $G$.

Given finite groups $G$ and $H$, by "a module ${ }_{G} X_{H}$ " is meant a $\mathbb{k}_{k} G-\mathbb{k}_{k} H$-bimodule, by "a module ${ }_{G} X$ " is meant a left $\mathbb{k}_{k} G$-module, and by "by a module $X_{H}$ is meant a right $\mathbb{k}_{\mathrm{k}} H$-module.

Given finite groups $G$ and $H$ and given a $\mathbb{k}_{k} G-\mathbb{k}_{k} H$-bimodule $X$, the dual vector space $X^{*}$ is a $\mathbb{k} H-\mathbb{k} G$-bimodule in a natural way $(h \cdot \phi \cdot g=\phi(g \cdot \bullet \cdot h)$ for all $g \in G, h \in H$ and $\left.\phi \in X^{*}\right)$. In particular, taking $H$ to be the trivial group, this defines a functor $X \mapsto X^{*}$ from left $\mathbb{k}_{k} G$-modules to right $\mathbb{k}_{k} G$-modules.

\subsection{Tensor product of duals and dual of a tensor product.}

Lemma 3.1. Let $H$ be a finite group with order not divisible by char $(\mathbb{k})$. For all finite dimensional $\mathbb{k}_{\mathrm{k}} H$-modules $X_{H}$ and ${ }_{H} Y$, there is a functorial bijective mapping

$$
\begin{aligned}
Y^{*} \otimes_{H} X^{*} & \rightarrow\left(X \otimes_{H} Y\right)^{*} \\
\phi_{2} \otimes \phi_{1} & \mapsto\left(x \otimes y \mapsto \sum_{h \in H} \phi_{1}(x \cdot h) \phi_{2}\left(h^{-1} \cdot y\right)\right) .
\end{aligned}
$$

Proof. The mapping is well defined. When $X=\mathbb{k}_{k} H_{H}$ and $Y={ }_{H} \mathbb{k} H$, it identifies with the following one, where $\left\{\delta_{h}\right\}_{h \in H}$ denotes the canonical basis of $\left(\mathbb{k}_{k} H\right)^{*}$,

$$
\begin{array}{cl}
\left(\mathbb{k}_{k} H\right)^{*} \otimes_{H}\left(\mathbb{k}_{k} H\right)^{*} & \rightarrow\left(\mathbb{k}_{k} H\right)^{*} \\
\delta_{h_{2}} \otimes \delta_{h_{1}} & \mapsto \delta_{h_{1} h_{2}} ;
\end{array}
$$

and the latter is bijective. Since $X_{H}$ and ${ }_{H} Y$ are finite dimensional and projective, then (3.1.1) is bijective.

The previous lemma generalises as follows. Given an integer $n \geqslant 2$, a sequence of groups $G_{0}, \ldots, G_{n}$ and a finite dimensional $\mathbb{k}_{i-1}-\mathbb{k}_{i} G_{i}$-bimodule $U_{i}$ for every $i \in\{1, \ldots, n\}$, there is a functorial bijective mapping

$$
X_{n}^{*} \otimes_{G_{n-1}} \cdots \otimes_{G_{1}} X_{1}^{*} \rightarrow\left(X_{1} \otimes_{G_{1}} \cdots \otimes_{G_{n-1}} X_{n}\right)^{*},
$$

which maps any $\phi_{n} \otimes \cdots \otimes \phi_{1}$ to the following linear form on $X_{1} \otimes_{G_{1}} \cdots \otimes_{G_{n-1}} X_{n}$,

$$
x_{1} \otimes \cdots \otimes x_{n} \mapsto \sum_{g_{1}, \ldots, g_{n-1}} \prod_{t=1}^{n} \phi_{t}\left(g_{t-1}^{-1} \cdot x_{t} \cdot g_{t}\right),
$$

where $g_{t}$ runs through $G_{t}(1 \leqslant t \leqslant n-1)$ and $g_{0}=g_{n}=e$. 


\subsection{Adjunctions.}

Lemma 3.2. Let $G$ and $H$ be finite groups with orders not divisible by char $(\mathbb{k})$. Let ${ }_{G} X_{H},{ }_{H} V$ and ${ }_{G} U$ be (bi)modules over $\mathbb{k}_{k} G$ and $\mathbb{k}_{k} H$ such that $X$ and $V$ are finite dimensional. Then, there exists a functorial isomorphism

$$
\Psi_{X}^{V, U}: \operatorname{Hom}_{H}\left(V, X^{*} \otimes_{G} U\right) \rightarrow \operatorname{Hom}_{G}\left(X \otimes_{H} V, U\right),
$$

such that, for all $\phi \in \operatorname{Hom}_{H}\left(V, X^{*} \otimes_{G} U\right), x \in X$ and $v \in V$,

$$
\left(\Psi_{X}^{V, U}(\phi)\right)(x \otimes v)=\sum_{g \in G}\left(\phi^{(2)}(v)\right)\left(g^{-1} \cdot x\right) g \cdot \phi^{(1)}(v),
$$

where the following notation is used with sum sign omitted, for all $v \in V$,

$$
\phi(v)=\phi^{(2)}(v) \otimes \phi^{(1)}(v) \in X^{*} \otimes_{G} U .
$$

In particular, the pair of functors $\left(X \otimes_{H}-, X^{*} \otimes_{G}-\right)$ between finite dimensional left $\mathbb{k}_{\mathrm{k} H}$-modules and finite dimensional left $\mathbb{k}_{\mathrm{k}} G$-modules is adjoint.

Proof. Using the definition of the structure of $H-G$-bimodule of $X^{*}$, simple changes of variable in the sum " $\sum_{g \in G}$ " show that $\Psi_{X}^{V, U}$ is indeed well defined.

Since ${ }_{H} V$ is finitely generated and projective, it suffices to prove the lemma assuming that $V=\mathbb{k}_{k} H$. In this case, $\Psi_{X}^{V, U}$ identifies with the mapping $\lambda_{X, U}$ defined (for all left $\mathbb{k}_{\mathrm{k}} G$-modules $X$ and $U$ ) by

$$
\begin{aligned}
X^{*} \otimes_{G} U & \rightarrow \operatorname{Hom}_{G}(X, U) \\
\phi \otimes u & \mapsto\left(x \mapsto \sum_{g \in G} \phi\left(g^{-1} \cdot x\right) g \cdot u\right) .
\end{aligned}
$$

Now, $\lambda_{\mathbb{k} G, \mathbb{k} G}$ identifies with the following bijective mapping (recall that $|G| \in \mathbb{k}^{\times}$).

$$
\begin{array}{ccc}
\mathbb{k}_{k} G^{*} \otimes_{G} \mathbb{k} G & \rightarrow & \mathbb{k}_{k} G \\
\phi \otimes e & \mapsto & \sum_{g \in G} \phi\left(g^{-1}\right) g .
\end{array}
$$

Since ${ }_{G} X$ is finite dimensional and projective, and since ${ }_{G} U$ is projective, it follows that $\lambda_{X, U}$ is bijective. Thus, $\Psi_{X}^{V, U}$ is bijective.

Lemma 3.3. Let $G, H, K$ be finite groups with orders not divisible by char $(\mathbb{k})$. Let ${ }_{G} X_{H},{ }_{H} Y_{K},{ }_{K} W$ and ${ }_{G} U$ be finite dimensional modules. Then, the following diagram commutes

$$
\begin{aligned}
& \operatorname{Hom}_{K}\left(W, Y^{*} \otimes_{H} X^{*} \otimes_{G} U\right) \longrightarrow \operatorname{Hom}_{K}\left(W,\left(X \otimes_{H} Y\right)^{*} \otimes_{G} U\right) \\
& \Psi_{Y}^{W, X^{*} \otimes_{G} U} \downarrow \downarrow_{\Psi_{X}^{W, U} \otimes_{H}^{Y}} \\
& \operatorname{Hom}_{H}\left(Y \otimes_{K} W, X^{*} \otimes_{G} U\right) \underset{\Psi_{X}^{Y \otimes_{K} W, U}}{\longrightarrow} \operatorname{Hom}_{G}\left(X \otimes_{H} Y \otimes_{K} W, U\right),
\end{aligned}
$$

where the top horizontal arrow is obtained upon applying $\operatorname{Hom}_{K}\left(W,-\otimes_{G} U\right)$ to (3.1.1).

Proof. In view of the compatibility of the involved mappings with direct sum decompositions of the finite dimensional projective modules ${ }_{K} W$ and ${ }_{G} U$, it is sufficient to prove the lemma assuming that $W=\mathbb{k} K$ and $U=\mathbb{k} G$. In this case, the given diagram identifies with the following one

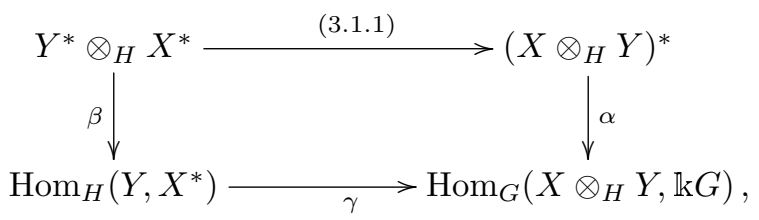

where, after using (3.2.2), 
- $\alpha$ is given by $\phi \mapsto\left(x \otimes y \mapsto \sum_{g \in G} \phi\left(g^{-1} \cdot x \otimes y\right) g\right)$,

- $\beta$ is given by $\phi_{2} \otimes \phi_{1} \mapsto(y \mapsto \sum_{h \in H} \phi_{2}\left(h^{-1} \cdot y\right) \underbrace{h \cdot \phi_{1}}_{\phi_{1}(\bullet h)})$ and

- $\gamma$ is given by $f \mapsto\left(x \otimes y \mapsto \sum_{g \in G}(f(y))\left(g^{-1} \cdot x\right) g\right)$.

It is direct to check that (3.2.4) commutes, which proves the lemma.

\subsection{Pairing of morphisms.}

Definition 3.4. Let $G$ and $H$ be finite groups whose orders are not divisible by $\operatorname{char}(\mathbb{k})$. Let ${ }_{G} X_{H},{ }_{H} V$, and ${ }_{G} U$ be (bi)modules such that $X$ and $V$ are finite dimensional. Assume that $U$ is simple. Define a pairing

$$
\langle-\mid-\rangle: \operatorname{Hom}_{G}\left(U, X \otimes_{H} V\right) \otimes_{\mathbb{k}} \operatorname{Hom}_{H}\left(V, X^{*} \otimes_{G} U\right) \rightarrow \mathbb{k}
$$

as follows. For all $f \in \operatorname{Hom}_{G}\left(U, X \otimes_{H} V\right)$ and $\phi \in \operatorname{Hom}_{H}\left(V, X^{*} \otimes_{G} U\right)$,

$$
\langle f \mid \phi\rangle \cdot \operatorname{Id}_{U}=\Psi_{X}^{V, U}(\phi) \circ f
$$

(following Lemma 3.2, the pairing $\langle-\mid-\rangle$ is non-degenerate).

This pairing is compatible with the following operation on morphisms.

Definition 3.5. Let $G, H$ and $K$ be finite groups whose orders are not divisible by $\operatorname{char}(\mathbb{k})$. Let ${ }_{G} X_{H},{ }_{H} Y_{K},{ }_{G} U,{ }_{H} V$ and ${ }_{K} W$ be (bi)modules. For all $f_{1} \in$ $\operatorname{Hom}_{G}\left(U, X \otimes_{H} V\right)$ and $f_{2} \in \operatorname{Hom}_{H}\left(V, Y \otimes_{K} W\right)$, define $f_{2} \times f_{1} \in \operatorname{Hom}_{G}\left(U, X \otimes_{H}\right.$ $\left.Y \otimes_{K} W\right)$ as the following composite morphism

$$
f_{2} \times f_{1}: U \stackrel{f_{1}}{\longrightarrow} X \otimes_{H} V \stackrel{\left(X \otimes_{H} f_{2}\right)}{\longrightarrow} X \otimes_{H} Y \otimes_{K} W .
$$

Here is the above-mentioned compatibility.

\section{Lemma 3.6. Let}

- $G, H$, and $K$ be finite groups with orders not divisible by $\operatorname{char}(\mathbb{k})$,

- ${ }_{G} X_{H},{ }_{H} Y_{K},{ }_{G} U,{ }_{H} V$ and ${ }_{K} W$ be (bi)modules such that $X, Y$, and $W$ are finite dimensional and $U$ and $V$ are simple,

- $f_{1} \in \operatorname{Hom}_{G}\left(U, X \otimes_{H} V\right)$ and $f_{2} \in \operatorname{Hom}_{H}\left(V, Y \otimes_{K} W\right)$ and

- $\phi_{1} \in \operatorname{Hom}_{H}\left(V, X^{*} \otimes_{G} U\right)$ and $\phi_{2} \in \operatorname{Hom}_{K}\left(W, Y^{*} \otimes_{H} V\right)$.

Denote by $\lambda$ the following isomorphism obtained upon applying $-\otimes_{G} U$ to (3.1.1),

$$
Y^{*} \otimes_{H} X^{*} \otimes_{G} U \stackrel{\sim}{\rightarrow}\left(X \otimes_{H} Y\right)^{*} \otimes_{G} U .
$$

Then, $\left\langle f_{2} \times f_{1} \mid \lambda \circ\left(\phi_{1} \times \phi_{2}\right)\right\rangle=\left\langle f_{2} \mid \phi_{2}\right\rangle \cdot\left\langle f_{1} \mid \phi_{1}\right\rangle$.

Proof. Using (3.2.3) yields that

$$
\Psi_{X \otimes_{H} Y}^{W, U}\left(\lambda \circ\left(\phi_{1} \times \phi_{2}\right)\right)=\Psi_{X}^{Y \otimes_{K} W, U} \circ \Psi_{Y}^{W, X^{*} \otimes_{G} U}\left(\phi_{1} \times \phi_{2}\right) .
$$

Recall that $\phi_{1} \times \phi_{2}$ is the following composite morphism

$$
W \stackrel{\phi_{2}}{\longrightarrow} Y^{*} \otimes_{H} V \stackrel{Y^{*} \otimes_{H} \phi_{1}}{\longrightarrow} Y^{*} \otimes_{H} X^{*} \otimes_{G} U .
$$

Since $\Psi_{X}^{\bullet \bullet \bullet}$ and $\Psi_{Y}^{\bullet \bullet \bullet}$ are adjunction isomorphisms, then

$$
\begin{aligned}
\Psi_{X}^{Y \otimes_{K} W, U} \circ \Psi_{Y}^{W, X^{*} \otimes_{G} U}\left(\phi_{1} \times \phi_{2}\right) & =\Psi_{X}^{Y \otimes_{K} W, U}\left(\phi_{1} \circ \Psi_{Y}^{W, V}\left(\phi_{2}\right)\right) \\
& =\Psi_{X}^{V, U}\left(\phi_{1}\right) \circ\left(X \otimes_{H} \Psi_{Y}^{W, V}\left(\phi_{2}\right)\right) .
\end{aligned}
$$

Therefore,

$$
\begin{aligned}
\Psi_{X \otimes_{H} Y}^{W, U}\left(\lambda \circ\left(\phi_{1} \times \phi_{2}\right)\right) \circ\left(f_{2} \times f_{1}\right)= & \Psi_{X}^{V, U}\left(\phi_{1}\right) \circ\left(X \otimes_{H} \Psi_{Y}^{W, V}\left(\phi_{2}\right)\right) \circ \\
& \left(X \otimes_{H} f_{2}\right) \circ f_{1} \\
= & \Psi_{X}^{V, U}\left(\phi_{1}\right) \circ\left(X \otimes_{H}\left\langle f_{2} \mid \phi_{2}\right\rangle \cdot \mathrm{Id}_{V}\right) \circ f_{1} \\
= & \left\langle f_{1} \mid \phi_{1}\right\rangle \cdot\left\langle f_{2} \mid \phi_{2}\right\rangle \cdot \operatorname{Id}_{U} .
\end{aligned}
$$

The conclusion of the lemma then follows from the definition of $\langle-\mid-\rangle$. 
The previous lemma has the following generalisation. Consider

- an integer $n \geqslant 2$,

- finite groups $G_{0}, \ldots, G_{n}$ with orders not divisible by $\operatorname{char}(\mathbb{k})$,

- a finite dimensional left $\mathbb{k}_{k} G_{i}$-module $U_{i}$ for every $i \in\{0, \ldots, n\}$, such that $U_{0}, \ldots, U_{n-1}$ are simple,

- a finite dimensional $\mathbb{k}_{k} G_{i-1}-\mathbb{k}_{k} G_{i}$-bimodule $X_{i}$ for every $i \in\{1, \ldots, n\}$,

- $f_{i} \in \operatorname{Hom}_{G_{i-1}}\left(U_{i-1}, X_{i} \otimes_{G_{i}} U_{i}\right)$ and $\phi_{i} \in \operatorname{Hom}_{G_{i}}\left(U_{i}, X_{i}^{*} \otimes_{G_{i}} U_{i-1}\right)$ for every $i \in\{1, \ldots, n\}$.

Denote by $\lambda^{(n)}$ the isomorphism obtained upon applying $-\otimes_{G_{0}} U_{0}$ to (3.1.2),

$$
\lambda^{(n)}: X_{n}^{*} \otimes_{G_{n-1}} \cdots \otimes_{G_{1}} X_{1}^{*} \otimes_{G_{0}} U_{0} \stackrel{\sim}{\longrightarrow}\left(X_{1} \otimes_{G_{1}} \cdots \otimes_{G_{n-1}} X_{n}\right)^{*} \otimes_{G_{0}} U_{0} .
$$

Then,

$$
\left\langle f_{n} \times \cdots \times f_{1} \mid \lambda^{(n)} \circ\left(\phi_{1} \times \cdots \times \phi_{n}\right)\right\rangle=\prod_{t=1}^{n}\left\langle f_{i} \mid \phi_{i}\right\rangle .
$$

\section{INTERTWINERS}

Let $Q_{0}, S, G$ and $M$ be as in Setting 1.1. This section establishes properties on intertwiners relative to $M$ which are needed to prove Theorem 1.25. This includes the properties of $\circledast$ mentioned in Section 1 as well as Proposition 1.20 on the existence of the pairing $(-\mid-)$. This is done in several steps,

- first, by proving that, in Definition 1.8 , the $\mathbb{k}_{k} G_{i_{0}}$-module $M(\underline{i} ; V)$ is isomorphic to $M(\underline{i}) \otimes_{G_{i_{n}}} V$ for a suitable $\mathbb{k}_{k} G_{i_{0}}-\mathbb{k}_{k} G_{i_{n}}$-bimodule $M(\underline{i})$;

- next, by proving that, under this isomorphism, $\circledast$ corresponds to the operation $\times$ of Definition 3.5;

- and finally by defining $(-\mid-)$ so that, under this isomorphism, it corresponds to the pairing $\langle-\mid-\rangle$ of Definition 3.4.

These steps are proceeded in turn in Sections 4.1, 4.2, and 4.3, respectively. They use the bimodule $M G$ defined as follows. Note that the action of $G$ on $S$ yields the skew group algebra $S * G$. Like in Section 3, for all groups $H$, the pieces of notation $e, \otimes_{H}$ and $\operatorname{Hom}_{H}$ stand for the neutral element of $H$, for $\otimes_{\mathbb{k} H}$, and for $\operatorname{Hom}_{\mathbb{k} H}$, respectively.

Definition 4.1. Define $M G$ as the following $S * G-S * G$-bimodule.

- Its underlying vector space is $M \otimes_{\mathrm{k}} \mathbb{k} G$, a tensor $m \otimes g$ is denoted by $m * g$ for all $m \in M$ and $g \in G$.

- The actions of $S$ and $G$ on the left and on the right are such that, for all $m \in M, g, h, k \in G$ and $s, s^{\prime} \in S$,

$$
\begin{aligned}
& s \cdot(m * g) \cdot s^{\prime}=s m{ }^{g} s^{\prime} * g \\
& h \cdot(m * g) \cdot k={ }^{h} m * h g k .
\end{aligned}
$$

The $S * G-S * G$-bimodule $M^{*} G$ is defined similarly after replacing $M$ by $M^{*}$.

4.1. The space $M(\underline{i})$. Let $n$ be a positive integer. Let $\underline{i}=i_{0}, \ldots, i_{n}$ a sequence in $Q_{0}$. Denote by $M(\underline{i})$ the following $\mathbb{k}_{k} G_{i_{0}}-\mathbb{k}_{k} G_{i_{n}}$-bimodule,

$$
M(\underline{i})=e_{i_{0}} M G e_{i_{1}} \otimes_{G_{i_{1}}} \cdots \otimes_{G_{i_{n-1}}} e_{i_{n-1}} M G e_{i_{n}} .
$$

Note that this yields the $\mathbb{k} G_{i_{n}}-\mathbb{k} G_{i_{0}}$-bimodule $M^{*}\left(\underline{i}^{o}\right)$. These bimodules are dual to each other. Here is an explicit isomorphism used later. Consider the isomorphism (3.1.2) taking $X_{t}=e_{i_{t-1}} M^{*} G e_{i_{t}}$ for all $t \in\{1, \ldots, n\}$,

$$
e_{i_{n}}(M G)^{*} e_{i_{n-1}} \otimes_{G_{i_{n-1}}} \cdots \otimes_{G_{i_{1}}} e_{i_{1}}(M G)^{*} e_{i_{0}} \stackrel{(3.1 .2)}{\longrightarrow} M(\underline{i})^{*} .
$$


Since the following mapping is a non-degenerate pairing

$$
\begin{aligned}
{[-\mid-]: \quad M G \otimes_{\mathbb{k}} M^{*} G } & \rightarrow \mathbb{k} \\
(m * g) \otimes(\varphi * h) & \mapsto \varphi\left({ }^{g^{-1}} m\right) \delta_{e, h g},
\end{aligned}
$$

it induces an isomorphism (of $S * G-S * G$-bimodules)

$$
\begin{aligned}
M^{*} G & \rightarrow(M G)^{*} \\
\bullet & \mapsto[-\mid \bullet]
\end{aligned}
$$

the $n$-th tensor power of which induces an isomorphism of $\mathbb{k} G_{i_{n}}-\mathbb{k} G_{i_{0}}$-bimodules,

$$
M^{*}\left(\underline{i}^{o}\right) \stackrel{\sim}{\longrightarrow} e_{i_{n}}(M G)^{*} e_{i_{n-1}} \otimes_{G_{i_{n-1}}} \cdots \otimes_{G_{i_{1}}} e_{i_{1}}(M G)^{*} e_{i_{0}} .
$$

Composing (4.1.2) and (4.1.4) yields an isomorphism of $\mathbb{k} G_{i_{n}}-\mathbb{k}_{k} G_{i_{0}}$-bimodules

$$
\Lambda_{\underline{i}}: M^{*}\left(\underline{i}^{o}\right) \stackrel{\sim}{\longrightarrow} M(\underline{i})^{*},
$$

which is induced by the mapping from $(M G)^{\otimes_{\mathrm{k}} n}$ to $\left((M G)^{\otimes_{\mathrm{k}} n}\right)^{*}$ which assigns to any tensor $\left(\varphi_{n} * g_{n}\right) \otimes \cdots \otimes\left(\varphi_{1} * g_{1}\right)$ the following linear form on $(M G)^{\otimes_{\mathrm{k}} n}$,

$$
\left(m_{1} * g_{1}^{\prime}\right) \otimes \cdots \otimes\left(m_{n} * g_{n}^{\prime}\right) \mapsto \sum_{k_{1}, \ldots, k_{n-1}} \prod_{t=1}^{n} \varphi_{t}\left({ }^{k_{t}^{-1} g_{t}^{\prime-1}} m_{t}\right) \delta_{e, g_{t} k_{t-1}^{-1} g_{t}^{\prime} k_{t}},
$$

where $\delta$ denotes the Kronecker symbol, $k_{t}$ runs through $G_{i_{t}}$ and $k_{n}=k_{0}=e$.

The bimodules $M(\underline{i})$ and $M^{*}\left(\underline{i}^{o}\right)$ are hence objects of the monoidal category $\left(\bmod \left(A^{e}\right), \otimes_{A}\right)$ in which they are left dual to each other. The following lemma describes tensor products of these objects in this monoidal category. The same statements are valid after replacing $M$ by $M^{*}$.

Lemma 4.2. Let $m, n$ be positive integers. Let $\underline{i^{\prime \prime}}=i_{0}, \ldots, i_{m+n}$ be a sequence in $Q_{0}$. Denote the sequences $i_{0}, \ldots, i_{m}$ and $i_{m}, \ldots, \bar{i}_{m+n}$ by $\underline{i}$ and $\underline{i}^{\prime}$, respectively.

(1) $M\left(\underline{i^{\prime \prime}}\right)=M(\underline{i}) \otimes_{G_{i_{m}}} M\left(\underline{i^{\prime}}\right)$.

(2) The following diagram is commutative

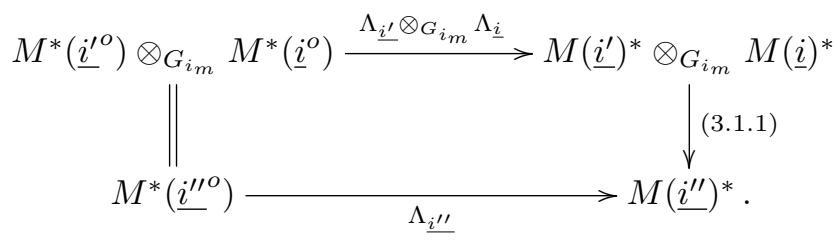

Proof. (1) follows from the definition of the spaces $M(\bullet)$ and (2) follows from the definition of the morphisms $\Lambda_{\bullet}$ in terms of (3.1.2) and (4.1.3).

The following result is the link between the bimodules $M(\bullet)$ and the modules of Definition 1.8. This link makes it possible to translate the results of Section 3 in terms of intertwiners. From now on, the piece of notation $*$ used for tensors in $M G$ is often dropped in order to avoid overloaded notation.

Lemma 4.3. Let $\underline{i}=i_{0}, \ldots, i_{n}$ be a sequence in $Q_{0}$, where $n \geqslant 1$. Let $U_{n} \in$ $\bmod \left(\mathbb{k}_{i_{n}}\right)$. There is an isomorphism in $\bmod \left(\mathbb{k}_{k} G_{i_{0}}\right)$,

$$
\Theta_{\underline{i} ; U_{n}}^{M}: M\left(\underline{i} ; U_{n}\right) \stackrel{\sim}{\longrightarrow} M(\underline{i}) \otimes_{G_{i_{n}}} U_{n}
$$

given by

$$
m_{1} \otimes \cdots \otimes m_{n} \otimes y_{1} \cdots y_{n} u \mapsto m_{1} y_{1} \otimes y_{1}^{-1} m_{2} y_{2} \otimes \cdots \otimes\left(y_{1} \cdots y_{n-1}\right)^{-1} m_{n} y_{n} \otimes u .
$$

When there is no risk of confusion, $\Theta_{\underline{i} ; U_{n}}^{M}$ is denoted by $\Theta_{\underline{i} ; U_{n}}$.

Proof. This is straightforward to check. 
The following natural transformation of functors from $\bmod \left(\mathbb{k} G_{i_{0}}\right)$ to $\bmod (\mathbb{k})$, defined by $\Theta_{\underline{i} ; U_{n}}$, is hence an isomorphism of functors,

$$
\begin{array}{ccc}
\operatorname{Hom}_{G_{i_{0}}}\left(-, M\left(\underline{i} ; U_{n}\right)\right) & \stackrel{\sim}{\longrightarrow} & \operatorname{Hom}_{G_{i_{0}}}\left(-, M(\underline{i}) \otimes_{G_{i_{n}}} U_{n}\right) \\
- & \longmapsto & \Theta_{\underline{i} ; U_{n}} \circ-.
\end{array}
$$

4.2. Compositions of intertwiners. It is now possible to compare the operation $\circledast$ on intertwiners, see Definition 1.13, and the operation $\times$ of Definition 3.5.

Lemma 4.4. Let $\underline{i}^{\prime \prime}=i_{0}, \ldots, i_{m+n}$ be a sequence in $Q_{0}$, where $m, n \geqslant 1$. Denote the sequences $i_{0}, \ldots, i_{m}$ and $i_{m}, i_{m+1}, \ldots, i_{m+n}$ by $\underline{i}$ and $\underline{i}^{\prime}$, respectively. Let $U_{0} \in \bmod \left(\mathbb{k}_{k} G_{i_{0}}\right), U_{m} \in \bmod \left(\mathbb{k}_{i_{m}}\right)$ and $U_{m+n} \in \bmod \left(\mathbb{k}_{i_{m+n}}\right)$. Finally, let $f \in \operatorname{Hom}_{\mathbb{k} G_{i_{0}}}\left(U_{0}, M\left(\underline{i} ; U_{m}\right)\right)$ and $f^{\prime} \in \operatorname{Hom}_{\mathbb{k} G_{i_{m}}}\left(U_{m}, M\left(\underline{i}^{\prime} ; U_{m+n}\right)\right)$. Then,

$$
\left(\Theta_{\underline{i^{\prime}} ; U_{m+n}} \circ f^{\prime}\right) \times\left(\Theta_{\underline{i} ; U_{m}} \circ f\right)=\Theta_{\underline{i^{\prime \prime} ; U_{m+n}}} \circ\left(f^{\prime} \circledast f\right) .
$$

As a consequence, $f^{\prime} \circledast f \in \operatorname{Hom}_{G_{i_{0}}}\left(U_{0}, M\left(\underline{i^{\prime \prime}} ; U_{m+n}\right)\right)$.

Proof. Let $u \in U_{0}$. Since $\Theta_{\underline{i^{\prime} ; U_{m+n}}} \circ f^{\prime}$ lies in $\operatorname{Hom}_{G_{i_{m}}}\left(U_{m}, M\left(\underline{i^{\prime \prime}}\right) \otimes_{G_{i_{m+n}}} U_{m+n}\right)$ and $\Theta_{\underline{i} ; U_{m}} \circ f$ lies in $\operatorname{Hom}_{G_{i_{0}}}\left(U_{0}, M(\underline{i}) \otimes_{G_{i_{m}}} U_{m}\right)$, then

$\left(\left(\Theta_{\underline{i}^{\prime} ; U_{m+n}} \circ f^{\prime}\right) \times\left(\Theta_{\underline{i} ; U_{m}} \circ f\right)\right)(u) \underset{(3.3 .3)}{=}\left(M(\underline{i}) \otimes_{\mathbb{k} G_{i_{m}}}\left(\Theta_{\underline{i}^{\prime} ; U_{m+n}} \circ f^{\prime}\right)\right) \circ\left(\Theta_{\underline{i} ; U_{m}} \circ f\right)(u)$.

Using the definition of $\Theta_{\underline{i} ; U_{m}}$, see (4.1.7), the right-hand side is equal to

$$
\begin{aligned}
& \left(M(\underline{i}) \otimes_{\mathbb{k} G_{i_{m}}}\left(\Theta_{\underline{\underline{\prime}}^{\prime} ; U_{m+n}} \circ f^{\prime}\right)\right)\left(\sum_{\underline{y}} f_{\underline{y}}^{(1)}(u) y_{1} \otimes y_{1}^{-1} f_{\underline{y}}^{(2)}(u) y_{2} \otimes \cdots\right. \\
& \left.\cdots \otimes\left(y_{1} \cdots y_{m-1}\right)^{-1}\left(f_{\underline{y}}^{(m)}(u)\right) y_{m} \otimes f_{\underline{y}}^{(0)}(u)\right),
\end{aligned}
$$

where $\underline{y}$ runs through all sequences $y_{1}, \ldots, y_{m}$ such that $y_{t} \in\left[G / G_{i_{t}}\right]$ for all $t \in$ $\{1, \ldots, m\}$. Using the definition of $\Theta_{i^{\prime} ; U_{m+n}}$, see (4.1.7), this is equal to

$$
\begin{aligned}
& \sum_{\underline{y}, \underline{y}^{\prime}} f_{\underline{y}}^{(1)}(u) y_{1} \otimes \cdots \otimes\left(y_{1} \cdots y_{m-1}\right)^{-1}\left(f_{\underline{y}}^{(m)}(u)\right) y_{m} \otimes \\
& f_{\underline{y}^{\prime}}^{\prime(\overline{1})}\left(f_{\underline{y}}^{(0)}(u)\right) y_{m+1} \otimes y_{m+1}^{-1}\left(f_{y^{\prime}}^{\prime(2)}\left(f_{\underline{y}}^{(0)}(u)\right)\right) y_{m+2} \otimes \cdots \\
& \cdots \otimes\left(y_{m+1} \cdots y_{m+n}\right)^{-1}\left(f_{\underline{y^{\prime}}}^{\prime(n)}\left(f_{\underline{y}}^{(0)}(u)\right)\right) y_{m+n} \otimes f_{\underline{y^{\prime}}}^{(0)}\left(f_{\underline{y}}^{(0)}(u)\right),
\end{aligned}
$$

where $y$ is as before and $y^{\prime}$ runs through all sequences $y_{m+1}, \ldots, y_{m+n}$ such that $y_{m+t} \in\left[G / G_{i_{m+t}}\right]$ for all $t \in\{1, \ldots, n\}$. Using the definition of $\Theta_{\underline{i}^{\prime \prime} ; U_{m+n}}$, see (4.1.7), this is equal to

$$
\begin{aligned}
& \Theta_{\underline{i^{\prime \prime} ; U_{m+n}}}\left(\sum_{\underline{y^{\prime \prime}}} f_{\underline{y}}^{(1)}(u) \otimes \cdots \otimes f_{\underline{y}}^{(m)}(u) \otimes y_{1} \cdots y_{m}\left(f_{y^{\prime}}^{\prime(1)}\left(f_{\underline{y}}^{(0)}(u)\right)\right) \otimes \cdots\right. \\
& \cdots \otimes y_{1} \cdots y_{m} \\
& \left.\left(f_{\underline{y^{\prime}}}^{\prime(n)}\left(f_{\underline{y}}^{(0)}(u)\right)\right) \otimes y_{1} \cdots y_{m+n} f_{\underline{y^{\prime}}}^{\prime(0)}\left(f_{\underline{y}}^{(0)}(u)\right)\right),
\end{aligned}
$$

where $y^{\prime \prime}$ runs through all sequences $y_{1}, \ldots, y_{m+n}$ such that $y_{t} \in\left[G / G_{i_{t}}\right]$ for all $t \in\{1, \ldots, m+n\}$ and where $\underline{y}$ and $y^{\prime}$ stand for the sequences $y_{1}, \ldots, y_{m}$ and $y_{m+1}, \ldots, y_{m+n}$, respectively. Finally, using the definition of $\circledast$, see (1.4.1), this is equal to $\left(\Theta_{\underline{i}^{\prime \prime} ; U_{m+n}} \circ\left(f^{\prime} \circledast f\right)\right)(u)$. Whence $(4.2 .1)$. Note that $f^{\prime} \circledast f$ is a morphism of $\mathbb{k}_{k} G_{i_{0}}$-modules because

- $\Theta_{\underline{i^{\prime \prime} ; U_{m+n}}}$ is an isomorphism of $\mathbb{k} G_{i_{0}}$-modules,

- $f$ and $\Theta_{i ; U_{m}}$ are morphisms of $\mathbb{k}_{k} G_{i_{0}}$-modules,

- $f^{\prime}$ and $\Theta_{\underline{i^{\prime} ; U_{m+n}}}$ are morphisms of $\mathbb{k}_{i_{m}}$-modules,

- and the operation "×" yields morphisms of $\mathbb{k} G_{i_{0}}$-modules (see (3.3.3))

Lemma 4.4 shows that Definition 1.15 yields a, possibly non-associative, algebra.

Proposition 4.5. Intw is an associative and unital algebra. 
Proof. Note that $\mathcal{I}$ ntw is unital. Indeed, for all $i \in\left[G \backslash Q_{0}\right]$ and $U \in \operatorname{irr}\left(G_{i}\right)$, denote by $\varepsilon_{i, U}$ the intertwiner $\operatorname{Id}_{U} \in \operatorname{Hom}_{\mathbb{k} G_{i}}(U, M(i ; U))$, recall that $M(i ; U)=U$. Then $\sum_{i, U} \varepsilon_{i, U}$ is a unity for $\circledast$.

In the framework of Definition 3.5, the equality $f_{3} \times\left(f_{2} \times f_{1}\right)=\left(f_{3} \times f_{2}\right) \times f_{1}$ holds whenever both sides make sense because of the associativity of the tensor product. In view of Lemma 4.4 , this entails that $\circledast$ is associative.

4.3. The pairing on intertwiners. Let $n$ be a positive integer, let $\underline{i}=i_{0}, \ldots, i_{n}$ be a sequence in $Q_{0}$ and let $U_{0}$ and $U_{n}$ be left modules over $\mathbb{k}_{k} G_{i_{0}}$ and $\mathbb{k}_{k} G_{i_{n}}$, respectively. To every $\varphi \in \operatorname{Hom}_{G_{i_{n}}}\left(U_{n}, M^{*}\left(\underline{i}^{o} ; U_{0}\right)\right)$ is associated a $\mathbb{k}$-linear mapping

$$
\Phi(\varphi): M\left(\underline{i} ; U_{n}\right) \rightarrow U_{0}
$$

defined as follows. For every sequence of representatives $y_{1}, \ldots, y_{n}\left(y_{t} \in\left[G / G_{i_{t}}\right]\right)$ there exist unique sequences

$$
\underline{x}=x_{n-1}, \ldots, x_{0}\left(x_{t} \in\left[G / G_{i_{t}}\right]\right), \text { and } h_{n-1}, \ldots, h_{0}\left(h_{t} \in G_{i_{t}}\right)
$$

such that, for all $t \in\{1, \ldots, n\}$,

$$
\left(y_{t} \cdots y_{n}\right)^{-1}=x_{n-1} \cdots x_{t-1} h_{t-1} ;
$$

these sequences are determined by a decreasing induction: $y_{n}^{-1}=x_{n-1} h_{n-1}$ and $h_{t} y_{t}^{-1}=x_{t-1} h_{t-1}$ for all $t \in\{1, \ldots, n-1\}$; then, for every $m_{1} \otimes \cdots \otimes m_{t} \otimes y_{1} \cdots y_{n} u$ lying in $M\left(\underline{i} ; U_{n}\right)$, define the element $\Phi(\varphi)\left(m_{1} \otimes \cdots \otimes m_{t} \otimes y_{1} \cdots y_{n} u\right)$ of $U_{0}$, using Notation 1.18 , as

$$
\prod_{t=1}^{n} \varphi_{(t)}^{\frac{x}{(u)}}\left({ }^{\left(y_{1} \cdots y_{n}\right)^{-1}} m_{t}\right) h^{h_{0}^{-1}}\left(\varphi_{(0)}^{\underline{x}}(u)\right) .
$$

This does make sense, because $m_{t} \in{ }_{y_{1} \cdots y_{t-1} \cdot i_{t-1}} M_{y_{1} \cdots y_{t} \cdot i_{t}}$, because $\varphi_{(t)}^{\frac{x}{(s}}$ is linear form on $x_{n-1} \cdots x_{t-1} \cdot i_{t-1} M_{x_{n-1} \cdots x_{t} \cdot i_{t}}$, and because of (4.3.1).

After appropriate identifications, $\Phi$ is related to the adjunction isomorphism $\Psi_{M(\underline{i})}^{\bullet, \bullet}$ of section 3.2 as explained in the following result. Recall that $\Psi_{M(\underline{i})}^{U_{n}, U_{0}}, \Theta_{\underline{i}^{\circ} ; U_{0}}^{M^{*}}$ and $\Lambda_{\underline{i}}$ are defined in (3.2.1), (4.1.7), and (4.1.5), respectively.

Lemma 4.6. Keep the previous setting. Denote by $\varphi^{\prime}$ the following composite morphism of left $\mathbb{k}_{\mathrm{k}} G_{i_{n}}$-modules,

$$
U_{n} \stackrel{\varphi}{\rightarrow} M^{*}\left(\underline{i}^{o} ; U_{0}\right) \stackrel{\Theta^{i^{o} ; U_{0}}}{\longrightarrow} M^{*}\left(\underline{i}^{o}\right) \otimes_{G_{i_{0}}} U_{0} \stackrel{\Lambda_{\underline{i}} \otimes_{G_{i_{0}}} U_{0}}{\longrightarrow} M(\underline{i})^{*} \otimes_{G_{i_{0}}} U_{0} .
$$

Then,

$$
\Phi(\varphi)=\Psi_{M(\underline{i})}^{U_{n}, U_{0}}\left(\varphi^{\prime}\right) \circ \Theta_{\underline{i} ; U_{n}}^{M} .
$$

In particular, $\Phi(\varphi) \in \operatorname{Hom}_{G_{i_{0}}}\left(M\left(\underline{i} ; U_{n}\right), U_{0}\right)$.

Proof. The composition $\Theta_{\underline{i}^{\circ} ; U_{0}}^{M^{*}} \circ \varphi$ is given by (see (4.1.7))

$u \mapsto \sum_{\underline{x}} \varphi_{(n)}^{\underline{x}}(u) x_{n-1} \otimes x_{n-1}^{-1} \varphi_{(n-1)}^{\underline{x}}(u) x_{n-2} \otimes \cdots \otimes\left(x_{n-1} \cdots x_{1}\right)^{-1} \varphi_{(1)}^{\underline{x}}(u) x_{0} \otimes \varphi_{(0)}^{\underline{x}}(u)$,

where $\underline{x}=x_{n-1}, \ldots, x_{0}$ runs through all sequences of representatives $\left(x_{t} \in\left[G / G_{i_{t}}\right]\right.$ for all $0 \leqslant t \leqslant n-1)$. Hence, following (4.1.6), the morphism $\varphi^{\prime}$ is given by

$$
u \mapsto \sum_{\underline{x}} \phi^{\underline{x}}(u) \otimes \varphi_{(0)}^{\underline{x}}(u)
$$


where $\phi^{\underline{x}}(u)$ is the linear form on $M(\underline{i})$, which is a quotient of $(M G)^{\otimes_{\mathrm{k}} n}$, induced by the linear form on $(M G)^{\otimes_{\mathrm{k}} n}$ mapping any $\left(m_{1} * g_{1}\right) \otimes \cdots \otimes\left(m_{n} * g_{n}\right)$ to

$$
\sum_{k_{1}, \ldots, k_{n-1}} \prod_{t=1}^{n}\left(\left(x_{n-1} x_{n-2} \cdots x_{t}\right)^{-1}\left(\varphi_{(t)}^{\underline{x}}(u)\right)\right)\left(k_{t}^{-1} g_{t}^{-1} m_{t}\right) \cdot \delta_{e, x_{t-1} k_{t-1}^{-1} g_{t} k_{t}},
$$

where $\delta$ denotes the Kronecker symbol, $k_{0}=k_{n}=e$ and $k_{t}$ runs through $G_{i_{t}}$ for $1 \leqslant t \leqslant n-1$.

In order to simplify the expression of (4.3.4), consider a tensor $\left(m_{1} * g_{1}\right) \otimes$ $\cdots \otimes\left(m_{n} * g_{n}\right)$ in $(M G)^{\otimes_{\mathrm{k}} n}$; for all $k_{1}, \ldots, k_{n-1}$ in $G$, the following assertions are equivalent, keeping $k_{0}=k_{n}=e$,

(i) $(\forall t \in\{1, \ldots, n-1\}) e=x_{t-1} k_{t-1}^{-1} g_{t} k_{t}$

(ii) $k_{t}=\left(x_{t-1} \cdots x_{1} x_{0} g_{1} g_{2} \cdots g_{t}\right)^{-1}$ for all $t \in\{1, \ldots, n\}$, and $g_{1} \cdots g_{n}$ is the inverse of $x_{n-1} \cdots x_{1} x_{0}$;

given that each $\left(x_{n-1} x_{n-2} \cdots x_{t}\right)^{-1}\left(\varphi^{\frac{x}{(t)}}(u)\right)$ in (4.3.4) is a linear form on $x_{t-1} i_{t-1} M_{i_{t}}$ and each $k_{t}$ is constrained to lie in $G_{i_{t}}$, then (4.3.4) is equal to

$$
\prod_{t=1}^{n}\left(\varphi^{\frac{x}{(t)}}(u)\right)\left({ }^{x_{n-1} \cdots x_{1} x_{0} g_{1} g_{2} \cdots g_{t-1}} m_{t}\right) \delta_{e, g_{1} g_{2} \cdots g_{n} x_{n-1} \cdots x_{1} x_{0}},
$$

which simplifies to

$$
\prod_{t=1}^{n}\left(\varphi_{(t)}^{\frac{x}{(u)}}(u)\right)\left(\left(g_{t} g_{t+1} \cdots g_{n}\right)^{-1} m_{t}\right) \delta_{\left(g_{1} g_{2} \cdots g_{n}\right)^{-1}, x_{n-1} \cdots x_{1} x_{0}} .
$$

Thus, the morphism $\varphi^{\prime}$ is given by $u \mapsto \sum_{\underline{x}} \phi^{\underline{x}}(u) \otimes \varphi_{(0)}^{\underline{x}}(u)$, where $\phi^{\underline{x}}(u)$ is the linear form on $M(\underline{i})$ induced by the mapping assigning (4.3.5) to any element $\left(m_{1} * g_{1}\right) \otimes \cdots \otimes\left(m_{n} * g_{n}\right)$ of $(M G)^{\otimes_{\mathrm{k}} n}$.

Hence (see Lemma 3.2), $\Psi_{M(\underline{i})}^{U_{n}, U_{0}}\left(\varphi^{\prime}\right)$ is the morphism from $M(\underline{i}) \otimes_{G_{i_{n}}} U_{n}$ to $U_{0}$ induced by the mapping from $(M G)^{\otimes_{\mathrm{k}} n} \otimes_{\mathrm{k}} U_{n}$ to $U_{0}$ which maps any $\left(m_{1} * g_{1}\right) \otimes$ $\cdots \otimes\left(m_{n} * g_{n}\right) \otimes u$ to

$$
\begin{aligned}
& \sum_{\underline{x}} \sum_{g \in G_{i_{0}}} \phi^{\underline{x}}(u)\left(g^{-1} m_{1} g_{1} \otimes m_{2} g_{2} \otimes \cdots \otimes m_{n} g_{n}\right){ }^{g}\left(\varphi_{(0)}^{\underline{x}}(u)\right) \\
= & \sum_{g, \underline{x}} \prod_{t=1}^{n} \varphi_{(t)}^{\underline{x}}(u)\left({ }^{\left(g_{t} \cdots g_{n}\right)^{-1}} m_{t}\right) \delta_{\left(g_{1} \cdots g_{n}\right)^{-1} g, x_{n-1} \cdots x_{0}}{ }^{g}\left(\varphi_{(0)}^{\underline{x}}(u)\right) .
\end{aligned}
$$

Now, let $m_{1} \otimes \cdots \otimes m_{n} \otimes y_{1} \cdots y_{n} u \in M\left(\underline{i} ; U_{n}\right)$. Recall that its image under $\Theta_{\underline{i} ; U_{n}}^{M}$ is $m_{1} y_{1} \otimes y_{1}^{-1} m_{2} y_{2} \otimes \cdots \otimes\left(y_{1} \cdots y_{n-1}\right)^{-1} m_{n} y_{n} \otimes u$ (see (4.1.7)). Hence, its image under $\Psi_{M(\underline{i})}^{U_{n}, U_{0}}\left(\varphi^{\prime}\right) \circ \Theta_{\underline{i} ; U_{n}}^{M}$ is

$$
\sum_{g, \underline{x}} \prod_{t=1}^{n} \varphi_{(t)}^{\frac{x}{(t)}}(u)\left(\left(y_{1} \cdots y_{n}\right)^{-1} m_{t}\right) \delta_{\left(y_{1} \cdots y_{n}\right)^{-1} g, x_{n-1} \cdots x_{0}}{ }^{g}\left(\varphi_{(0)}^{\underline{x}}(u)\right) .
$$

Note that, given $g \in G_{i_{0}}$ and $\underline{x}$, then, for all $t \in\{1, \ldots, n\}$,

- $\varphi_{(t)}^{\underline{x}}(u) \in\left({ }_{x_{n-1} \cdots x_{t-1} \cdot i_{t-1}} M_{x_{n-1} \cdots x_{t} \cdot i_{t}}\right)^{*}$ and

- $\left(y_{1} \cdots y_{n}\right)^{-1} m_{t} \in\left(y_{t} \cdots y_{n}\right)^{-1 \cdot i_{t-1}} M_{\left(y_{t+1} \cdots y_{n}\right)^{-1} \cdot i_{t}}$;

in particular, if for a given index $(g, \underline{x})$ in (4.3.6) the product " $\prod_{t=1}^{n}$ " is non-zero then this product is the unique non-zero term of the sum (4.3.6). More precisely, let $\left\{x_{t}, h_{t}\right\}_{0 \leqslant t \leqslant n-1}$ be the unique collection of elements in $G$ such that, for all $t \in\{0, \ldots, n-1\}$,

- $x_{t} \in\left[G / G_{i_{t}}\right]$,

- $h_{t} \in G_{i_{t}}$ and

- $\left(y_{t+1} \cdots y_{n}\right)^{-1}=x_{n-1} \cdots x_{t} h_{t}$; 
then, the only possibly non-zero term of the sum (4.3.6) is the one with index $\left(h_{0}^{-1}, \underline{x}\right)$. Therefore, (4.3.6) equals

$$
\prod_{t=1}^{n} \varphi_{(t)}^{\frac{x}{(u)}}(u)\left({ }^{\left(y_{1} \cdots y_{n}\right)^{-1}} m_{t}\right)^{h_{0}^{-1}}\left(\varphi_{(0)}^{\underline{x}}(u)\right)
$$

which is equal to $\Phi(\varphi)\left(m_{1} \otimes \cdots \otimes m_{n} \otimes y_{1} \cdots y_{n} u\right)$ (see (4.3.2)). Thus, $\Phi(\varphi)=$ $\Psi_{M(\underline{i})}^{U_{n}, U_{0}}\left(\varphi^{\prime}\right) \circ \Theta_{\underline{i} ; U_{n}}^{M}$. This proves (4.3.3). The second assertion of the lemma is a direct consequence of (4.3.3).

It follows from Lemma 4.6 that the following mapping is bijective,

$$
\Phi: \operatorname{Hom}_{G_{i_{n}}}\left(U_{n}, M^{*}\left(\underline{i}^{o} ; U_{0}\right)\right) \rightarrow \operatorname{Hom}_{G_{i_{0}}}\left(M\left(\underline{i} ; U_{n}\right), U_{0}\right) .
$$

This yields a non-degenerate pairing defined as follows.

Definition 4.7. Let $\underline{i}=i_{0}, \ldots, i_{n}$ be a sequence in $Q_{0}$, where $n \geqslant 1$. Let $U_{0} \in$ $\bmod \left(\mathbb{k}_{k} G_{i_{0}}\right)$ be simple. Let $U_{n} \in \bmod \left(\mathbb{k}_{k} G_{i_{n}}\right)$. Define $(-\mid-)$ to be the non-degenerate pairing

$$
(-\mid-): \operatorname{Hom}_{G_{i_{0}}}\left(U_{0}, M\left(\underline{i} ; U_{n}\right)\right) \otimes_{\mathbb{k}} \operatorname{Hom}_{G_{i_{n}}}\left(U_{n}, M^{*}\left(\underline{i}^{o} ; U_{0}\right)\right) \rightarrow \mathbb{k},
$$

such that, for all $f \in \operatorname{Hom}_{G_{i_{0}}}\left(U_{0}, M\left(\underline{i} ; U_{n}\right)\right)$ and $\varphi \in \operatorname{Hom}_{G_{i_{n}}}\left(U_{n}, M^{*}\left(\underline{i}^{o}, U_{0}\right)\right)$,

$$
(f \mid \varphi) \cdot \operatorname{Id}_{U_{0}}=\Phi(\varphi) \circ f
$$

With this definition, it follows from (3.3.2) and (4.3.3) that

$$
(f \mid \varphi)=\left\langle\Theta_{\underline{i} ; U_{n}}^{M} \circ f \mid\left(\Lambda_{\underline{i}} \otimes_{G_{i_{0}}} U_{0}\right) \circ \Theta_{\underline{i}^{\circ} ; U_{0}}^{M^{*}} \circ \varphi\right\rangle .
$$

It is now possible to prove Proposition 1.20.

Proof of Proposition 1.20. Note that (1.5.2) is equal to $(\Phi(\varphi))(f(u))$, see (4.3.2). Hence, the pairing $(-\mid-)$ claimed by the proposition is the one of Definition 4.7.

The pairing $(-\mid-)$ is compatible with the composition of (dual) intertwiners in the following sense.

Lemma 4.8. Let $m, n$ be positive integers, let $\underline{i}^{\prime \prime}=i_{0}, \ldots, i_{m+n}$ be a sequence in $Q_{0}$, let $U_{0}$ and $U_{m}$ be simple left modules over $\mathbb{k}_{k} G_{i_{0}}$ and $\mathbb{k}_{k} G_{i_{m}}$, respectively, and let $U_{m+n}$ be a left $\mathbb{k}_{k} G_{i_{m+n}}$-module. Then, for all intertwiners

$$
\begin{array}{ll}
f_{1}: U_{0} \rightarrow M\left(\underline{i} ; U_{m}\right), & \varphi_{1}: U_{m} \rightarrow M^{*}\left(\underline{i}^{o} ; U_{0}\right), \\
f_{2}: U_{m} \rightarrow M\left(\underline{i^{\prime}} ; U_{m+n}\right), & \varphi_{2}: U_{m+n} \rightarrow M^{*}\left(\underline{i}^{\prime} ; U_{m}\right),
\end{array}
$$

where $\underline{i}=i_{0}, \ldots, i_{m}$ and $\underline{i^{\prime}}=i_{m}, \ldots, i_{m+n}$, the following holds

$$
\left(f_{2} \circledast f_{1} \mid \varphi_{1} \circledast \varphi_{2}\right)=\left(f_{2} \mid \varphi_{2}\right) \cdot\left(f_{1} \mid \varphi_{1}\right) .
$$

Proof. In order to avoid overloaded expressions, the following shorthand notation is used in this proof: $\Theta_{\underline{i} ; U_{0}}$ for $\Theta_{\underline{i} ; U_{0}}^{M}$ and $\Theta_{\underline{i} ; U_{0}}^{\prime}$ for $\Theta_{\underline{i}^{\circ} ; U_{0}}^{M^{*}}$. Define $f_{1}^{\prime}, f_{2}^{\prime}, \varphi_{1}^{\prime}, \varphi_{2}^{\prime}$ as follows

$$
\begin{array}{ll}
f_{1}^{\prime}=\Theta_{\underline{i} ; U_{m}} \circ f_{1}, & \varphi_{1}^{\prime}=\left(\Lambda_{\underline{i}} \otimes_{G_{i_{0}}} U_{0}\right) \circ \Theta_{\underline{i} ; U_{0}}^{\prime} \circ \varphi_{1}, \\
f_{2}^{\prime}=\Theta_{\underline{i^{\prime} ;} U_{m+n}} \circ f_{2}, & \varphi_{2}^{\prime}=\left(\Lambda_{\underline{i^{\prime}}} \otimes_{G_{i_{m}}} U_{m}\right) \circ \Theta_{\underline{i^{\prime} ; U_{m}}}^{\prime} \circ \varphi_{2} .
\end{array}
$$

It follows from (4.3.8) that $\left(f_{2} \circledast f_{1} \mid \varphi_{1} \circledast \varphi_{2}\right)$ is equal to

$$
\left\langle\Theta_{\underline{i^{\prime \prime}}, U_{m+n}} \circ\left(f_{2} \circledast f_{1}\right) \mid\left(\Lambda_{\underline{i^{\prime \prime}}} \otimes_{G_{i_{0}}} U_{0}\right) \circ \Theta_{\underline{i^{\prime \prime}, U_{0}}}^{\prime} \circ\left(\varphi_{1} \circledast \varphi_{2}\right)\right\rangle ;
$$

applying Lemma 4.4 twice, first for $M$ and next for $M^{*}$, yields that the previous expression is equal to

$$
\langle\underbrace{\left(\Theta_{\underline{i}^{\prime}, U_{m+n}} \circ f_{2}\right)}_{f_{1}^{\prime}} \times \underbrace{\left(\Theta_{\underline{\underline{i}}, U_{m}} \circ f_{1}\right)}_{f_{2}^{\prime}} \mid\left(\Lambda_{\underline{\underline{i}^{\prime \prime}}} \otimes_{G_{i_{0}}} U_{0}\right) \circ\left[\left(\Theta_{\underline{\underline{i}}, U_{0}}^{\prime} \circ \varphi_{1}\right) \times\left(\Theta_{\underline{i^{\prime}, U_{m}}}^{\prime} \circ \varphi_{2}\right)\right]\rangle ;
$$


according to Lemma 4.2 this is equal to

$$
\left\langle f_{1}^{\prime} \times f_{2}^{\prime} \mid \lambda \circ\left(\Lambda_{\underline{i^{\prime}}} \otimes_{G_{i_{m}}} \Lambda_{\underline{i}} \otimes_{G_{i_{0}}} U_{0}\right) \circ\left[\left(\Theta_{\underline{i}, U_{0}}^{\prime} \circ \varphi_{1}\right) \times\left(\Theta_{\underline{i}^{\prime}, U_{m}}^{\prime} \circ \varphi_{2}\right)\right]\right\rangle,
$$

where $\lambda$ is as in Lemma 3.6. Now, since the following composite morphisms are equal due to the associativity of the tensor product

$$
M^{*}\left(\underline{(i)}^{\prime o}\right) \underset{G_{i_{m}}}{\otimes} U_{m} \stackrel{1 \otimes\left(\Theta_{\underline{i} ; U_{0}}^{\prime} \circ \varphi_{1}\right)}{\longrightarrow} M^{*}\left(\underline{(i)}^{\prime o}\right) \underset{G_{i_{m}}}{\otimes} M^{*}\left(\underline{i}^{o}\right) \underset{G_{i_{0}}}{\otimes} U_{0} \stackrel{\Lambda_{\underline{i}^{\prime}} \otimes \Lambda_{\underline{i}} \otimes 1}{\longrightarrow} M\left(\underline{i}^{\prime}\right)^{*} \underset{G_{i_{m}}}{\otimes} M(\underline{i})^{*} \underset{G_{i_{0}}}{\otimes} U_{0}
$$

and

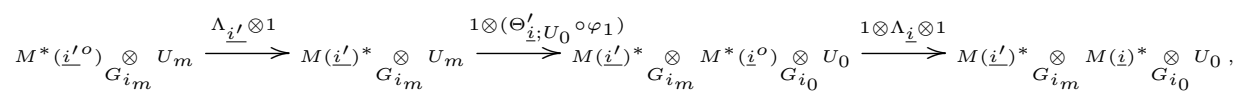

then $\left(\Lambda_{\underline{i^{\prime}}} \otimes_{G_{i_{m}}} \Lambda_{\underline{i}} \otimes_{G_{i_{0}}} U_{0}\right) \circ\left[\left(\Theta_{\underline{i}, U_{0}}^{\prime} \circ \varphi_{1}\right) \times\left(\Theta_{\underline{i}^{\prime}, U_{m}}^{\prime} \circ \varphi_{2}\right)\right]$ is equal to

$$
[\underbrace{\left(\Lambda_{\underline{i}} \otimes_{G_{i_{0}}} U_{0}\right) \circ \Theta_{\underline{i} ; U_{0}}^{\prime} \circ \varphi_{1}}_{\varphi_{1}^{\prime}}] \times[\underbrace{\left(\Lambda_{\underline{i^{\prime}}} \otimes_{G_{i_{m}}} U_{m}\right) \circ \Theta_{\underline{i}^{\prime} ; U_{m}}^{\prime} \circ \varphi_{1}}_{\varphi_{2}^{\prime}}] .
$$

Therefore,

$$
\begin{array}{cl}
= & \left\langle f_{2}^{\prime} \times f_{1}^{\prime} \mid \lambda \circ\left(\varphi_{1}^{\prime} \times \varphi_{2}^{\prime}\right)\right\rangle \\
= & \left\langle f_{2}^{\prime} \mid \varphi_{2}^{\prime}\right\rangle \cdot\left\langle f_{1}^{\prime} \mid \varphi_{1}^{\prime}\right\rangle \\
\overline{=} \begin{array}{cl}
\text { Lema 3.6 } \\
\overline{=}
\end{array} & \left(f_{2} \mid \varphi_{2}\right) \cdot\left(f_{1} \mid \varphi_{1}\right) .
\end{array}
$$

Thus, $\left(f_{2} \circledast f_{1} \mid \varphi_{1} \circledast \varphi_{2}\right)=\left(f_{1} \mid \varphi_{1}\right) \cdot\left(f_{2} \mid \varphi_{2}\right)$.

\section{Proof of the MAIN RESUlts}

This section proves Theorems 1.25 and 1.27. Keeping $Q_{0}, S, G$ and $M$ as in Setting 1.1 and $Q_{G}$ as in Setting 1.4, the proofs use the following notation:

- denote by $\hat{e}$ the idempotent $\sum_{i \in\left[G \backslash Q_{0}\right]} e_{i}$ of $S$, this idempotent is not to be confused with $\tilde{e}$ which is equal to $\sum_{(i, U)} e_{i} * \varepsilon_{U}$, where $(i, U)$ runs through the vertices of $Q_{G}$;

- denote by $S_{1}$ the $\mathbb{k}$-algebra $\Pi_{i \in\left[G \backslash Q_{0}\right]} \mathbb{k} G_{i}$.

First, it is necessary to check that the quiver $Q_{G}$ corresponds to the one given by Demonet in [3].

Proof of Theorem 1.6. Following [3], see p. 1057, the algebra $T_{S}(M) * G$ is Morita equivalent to $\tilde{e} \cdot\left(T_{S}(M) * G\right) \cdot \tilde{e}$, which is isomorphic to the path algebra of a quiver having the same vertices as $Q_{G}$ and such that the arrows from a vertex $(i, U)$ to a vertex $(j, V)$ form a basis of the vector space $\left(e_{i} * \varepsilon_{U}\right) \cdot(M G) \cdot\left(e_{j} * \varepsilon_{V}\right)$. Now, considering $e_{i}, e_{j}, \varepsilon_{U}$, and $\varepsilon_{V}$ as elements of $S * G$,

$$
\begin{aligned}
& \left(e_{i} * \varepsilon_{U}\right) \cdot(M G) \cdot\left(e_{j} * \varepsilon_{V}\right)=\varepsilon_{U} \cdot\left(e_{i} \cdot(M G) \cdot e_{j}\right) \cdot \varepsilon_{V} \\
& \underset{(4.1 .1)}{=} \quad \varepsilon_{U} \cdot M(i, j) \cdot \varepsilon_{V} \\
& \simeq \operatorname{Hom}_{\mathbb{k} G_{i}}\left(U, M(i, j) \otimes_{G_{j}} V\right) \\
& \underset{(4.1 .7)}{\simeq} \operatorname{Hom}_{\mathbb{k} G_{i}}(U, M(i, j ; V)) \text {. }
\end{aligned}
$$

Whence the first statement of the theorem. Now, if $U=\mathbb{k}_{k} G_{i} \cdot \varepsilon_{U}$ for all $(i, U) \in Q_{G}$, then $f\left(\varepsilon_{U}\right)$ does belong to $\left(e_{i} * \varepsilon_{U}\right) \cdot(M G) \cdot\left(e_{j} * \varepsilon_{V}\right)$, which is contained in $\tilde{e} \cdot\left(T_{S}(M) *\right.$ $G) \cdot \tilde{e}$, for all arrows $f:(i, U) \rightarrow(j, V)$ of $Q_{G}$. Whence the isomorphism (1.1.1).

The proof of Theorem 1.25 uses Morita equivalences in the graded sense. Two $\left(\mathbb{N}\right.$-)graded finite dimensional $\mathbb{k}$-algebras $\Lambda_{1}$ and $\Lambda_{2}$ are Morita equivalent in the graded sense if $\Lambda_{2} \simeq \operatorname{End}_{\Lambda_{1}}(P)^{\text {op }}$ for some graded projective $\Lambda_{1}$-module $P$ which is a generator of the category of graded $\Lambda_{1}$-modules, and hence of $\bmod \left(\Lambda_{1}\right)$. Both 
$\Lambda_{1}$ and $\Lambda_{2}$ are isomorphic as graded algebras to basic graded finite dimensional algebras; moreover $\Lambda_{1}$ and $\Lambda_{2}$ are Morita equivalent in the graded sense if and only if their basic versions are isomorphic as graded algebras.

Proof of Theorem 1.25. Denote by $\Phi$ the algebra homomorphism $\mathbb{k}_{k} Q_{G} \rightarrow \mathcal{I}_{\mathrm{ntw}}{ }^{\text {op }}$ given in the statement of the theorem. Hence, $\Phi(\gamma)=f_{\gamma}$ for all paths $\gamma$ in $Q_{G}$.

(1) Let $\gamma$ and $\gamma^{\prime}$ be parallel paths in $Q_{G}$. It follows from the definition of $\varphi_{\gamma^{\prime}}$, see Notation 1.23, and from (4.3.9) that

$$
\left(f_{\gamma} \mid \varphi_{\gamma^{\prime}}\right)= \begin{cases}1 & \text { if } \gamma=\gamma^{\prime} \\ 0 & \text { otherwise }\end{cases}
$$

Since $\Phi$ maps non parallel paths in $Q_{G}$ into distinct components of $\mathcal{I}$ ntw in the decomposition (1.4.3), then $\Phi$ is injective. In order to prove that $\Phi$ is an isomorphism, it is hence sufficient to prove that, for all integers $n$, the homogeneous components of degree $n$ of the graded algebras $\mathbb{k} Q_{G}$ and $\mathcal{I}$ ntw have the same dimensions. Note that $\mathbb{k}_{Q_{G}}$ is graded by the length of the paths in $Q_{G}$ and $\mathcal{I}$ ntw is graded so that every intertwiner lying in a space of the shape $\operatorname{Hom}_{\mathbb{k} G_{i_{0}}}\left(U, M\left(i_{0}, \ldots, i_{n} ; V\right)\right)$ is homogeneous of degree $n$.

First, following [3], see Theorem 1.6, the algebras $\mathbb{k}_{k} Q_{G}$ and $\tilde{e} \cdot\left(T_{S}(M) * G\right) \cdot \tilde{e}$ are isomorphic $\mathbb{k}$-algebras both Morita equivalent to $T_{S}(M) * G$. Actually, the proof given in [3] works in the graded setting.

Next, $\hat{e}$ may be considered as an idempotent of $T_{S}(M) * G$. In this sense,

$$
\tilde{e} \cdot\left(T_{S}(M) * G\right) \cdot \tilde{e}=\tilde{e} \cdot \hat{e}\left(T_{S}(M) * G\right) \cdot \hat{e} \cdot \tilde{e} .
$$

Following [3], see p. 1057, the algebra $\hat{e} \cdot\left(T_{S}(M) * G\right) \cdot \hat{e}$ is Morita equivalent to $T_{S_{1}}(\hat{e} \cdot(M G) \cdot \hat{e})$. Again, this is still true in the graded sense. Hence, $\tilde{e} \cdot\left(T_{S}(M) * G\right) \cdot \tilde{e}$, which is basic, is isomorphic to $\tilde{e} \cdot T_{S_{1}}(\hat{e} \cdot(M G) \cdot \hat{e}) \cdot \tilde{e}$ as a graded algebra.

Finally, by definition of $\tilde{e}$ and $\hat{e}$, the homogeneous component of degree $n$ of $\tilde{e} \cdot T_{S_{1}}(\hat{e} \cdot(M G) \cdot \hat{e}) \cdot \tilde{e}$ is equal to

$$
\bigoplus_{i_{0}, i_{n} \in\left[G \backslash Q_{0}\right], U_{0} \in \operatorname{irr}\left(G_{i_{0}}\right), U_{n} \in \operatorname{irr}\left(G_{i_{n}}\right)}\left(e_{i_{0}} * \varepsilon_{U_{0}}\right) \cdot(M G)^{\otimes_{S_{1}} n} \cdot\left(e_{i_{n}} * \varepsilon_{U_{n}}\right) .
$$

This is equal to

$$
\bigoplus_{i_{0}, \ldots, i_{n} \in\left[G \backslash Q_{0}\right], U_{0} \in \operatorname{irr}\left(G_{i_{0}}\right), U_{n} \in \operatorname{irr}\left(G_{i_{n}}\right)} \varepsilon_{U_{0}} \cdot M\left(i_{0}, \ldots, i_{n}\right) \cdot \varepsilon_{U_{n}} .
$$

This is isomorphic to

$$
\bigoplus_{i_{0}, \ldots, i_{n}, U_{0}, U_{n}} \operatorname{Hom}_{\mathbb{k} G_{i_{0}}}(U_{0}, \underbrace{M\left(i_{0}, \ldots, i_{n}\right) \otimes_{G_{i_{n}}} V}_{\simeq M\left(i_{0}, \ldots, i_{n} ; V\right)}) .
$$

These considerations prove that the homogeneous components of degree $n$ of $\mathbb{k} Q_{G}$ and $\mathcal{I}$ ntw are isomorphic. Thus, $\Phi$ is an isomorphism.

(2) follows from (5.0.1) and from the fact that $\Phi$ is surjective.

Proof of Theorem 1.2\%. First, $\Xi$ is bijective. Indeed, on one hand, there is an equality of vector subspaces of $T_{S}(M) * G$,

$\hat{e} \cdot\left(T_{S}(M) * G\right) \cdot \hat{e}=\bigoplus_{\underline{i}, \underline{y}}\left({ }_{i_{0}} M_{y_{1} \cdot i_{1}} \otimes_{\mathbb{k}} \cdots \otimes_{\mathbb{k}} y_{1} \cdots y_{n-1} \cdot i_{n-1} M_{y_{1} \cdots y_{n} \cdot i_{n}}\right) * y_{1} \cdots y_{n} \mathbb{k} G$,

where $\underline{i}$ runs through all sequences $i_{0}, \ldots, i_{n}$ of $\left[G \backslash Q_{0}\right]$ and $y$ runs through all sequences $y_{1}, \ldots, y_{n}$ such that $y_{t} \in\left[G / G_{i_{t}}\right]$ for all $t \in\{1, \ldots, n\}$. On the other 
hand, for all vertices $(i, U)$ and $(j, V)$ of $Q_{G}$ and for all $\mathbb{k}_{k} G_{i}-\mathbb{k}_{k} G_{j}$-bimodules $N$, the following mapping is bijective,

$$
\operatorname{Hom}_{\mathrm{k} G_{i}}\left(U, N \cdot \varepsilon_{V}\right) \longrightarrow \varepsilon_{U} \cdot N \cdot \varepsilon_{V}
$$

Hence, $\Xi$ is bijective and $\Xi^{-1}$ is given by $\Xi^{-1}(f)=f\left(\varepsilon_{U}\right)$ for all $f \in \mathcal{I}$ ntw with domain denoted by $U$.

Next, $\Xi$ is a morphism of $\mathbb{k}$-algebras. Indeed, for all $f \in \operatorname{Hom}_{\mathbb{k} G_{i_{0}}}(U, M(\underline{i} ; V))$ lying in $\mathcal{I}$ ntw, then $\Xi^{-1}(f)=f\left(\varepsilon_{U}\right)$. Therefore, with the setting of Definition 1.13, then $\left(f^{\prime} \circledast f\right)\left(\varepsilon_{U}\right)$ is equal to the product $f\left(\varepsilon_{V}\right) \cdot f^{\prime}\left(\varepsilon_{U}\right)$ in $T_{S}(M) * G$, see (1.4.1). Accordingly, $\Xi^{-1}$, and hence $\Xi$, is a $\mathbb{k}$-algebra homomorphism.

Finally, the diagram (1.6.4) is commutative. Indeed, all its arrows are $\mathbb{k}$-algebra homomorphisms. Hence, it suffices to prove the commutativity on the arrows of $Q_{G}$, which follows from the definitions of $\Xi,(1.1 .1)$, and (1.6.1). Note that (1.6.5) follows from (1.6.2).

\section{ACKNOWLEDGEMENTS}

I thank Edson Ribeiro Alvares for helpful comments on previous versions of this article. I also thank the referee for several suggestions that improved the presentation of the article.

\section{REFERENCES}

[1] C. Amiot and P.-G. Plamondon. The cluster category of a surface with punctures via group actions. arXiv:1707.01834 [math.RT], 2017.

[2] R. Bocklandt, T. Schedler, and M. Wemyss. Superpotentials and higher order derivations. J. Pure Appl. Algebra, 214(9):1501-1522, 2010.

[3] L. Demonet. Skew group algebras of path algebras and preprojective algebras. J. Algebra, 323(4):1052-1059, 2010

[4] J. Dionne, M. Lanzilotta, and D. Smith. Skew group algebras of piecewise hereditary algebras are piecewise hereditary. J. Pure Appl. Algebra, 213(2):241-249, 2009.

[5] P. Etingof, S. Gelaki, D. Nikshych, and V. Ostrik. Tensor categories, volume 205 of Mathematical Surveys and Monographs. American Mathematical Society, Providence, RI, 2015.

[6] V. Ginzburg. Calabi-Yau algebras. arXiv:math/0612139 v3, 2006.

[7] S. Giovannini. Higher representation infinite algebras from McKay quivers of metacyclic groups. Comm. Algebra, 47(9):3672-3711, 2019.

[8] S. Giovannini and A. Pasquali. Skew group algebras of Jacobian algebras. J. Algebra, 526:112$165,2019$.

[9] S. Giovannini, A. Pasquali, and P.-G. Plamondon. Quivers with potentials and actions of finite abelian groups. arXiv:1912.11284 [math.RT], 2019.

[10] P. Le Meur. Crossed-products of Calabi-Yau algebras by finite groups. Journal of Pure and Applied Algebra, 224(10):106394, 2020.

[11] L. Li. Finitistic dimensions and piecewise hereditary property of skew group algebras. Glasg. Math. J., 57(3):509-517, 2015.

[12] R. Martínez-Villa. Skew group algebras and their Yoneda algebras. Math. J. Okayama Univ., 43:1-16, 2001.

[13] I. Reiten and C. Riedtmann. Skew group algebras in the representation theory of Artin algebras. J. Algebra, 92(1):224-282, 1985.

[14] I. Reiten and M. Van den Bergh. Two-dimensional tame and maximal orders of finite representation type. Mem. Amer. Math. Soc., 80(408):viii+72, 1989.

Université de Paris, Sorbonne Université, CNRS, Institut de Mathématiques de Jussieu-Paris Rive Gauche, F-75013 Paris, France

Email address: patrick.le-meur@imj-prg.fr 